\title{
Simulated Annealing based Symbiotic Organisms Search Optimization Algorithm for Traveling Salesman Problem
}

\author{
Absalom El-Shamir Ezugwu ${ }^{1 *}$, Aderemi Oluyinka Adewumi ${ }^{1}$, Marc Eduard Frîncu ${ }^{2}$ \\ ${ }^{*}$ School of Mathematics, Statistics and Computer Science, University of Kwazulu-Natal, Westville Campus, \\ Private Bag X54001, Durban 4000, South Africa \\ ${ }^{2}$ Faculties of Mathematics and Computer Science, West University of Timisoara, Timisoara, Romania
}

ezugwua@ukzn.ac.za, adewumia@ukzn.ac.za,marc.frincu@e-uvt.ro

\begin{abstract}
Symbiotic Organisms Search (SOS) algorithm is an effective new metaheuristic search algorithm, which has recently recorded wider application in solving complex optimization problems. SOS mimics the symbiotic relationship strategies adopted by organisms in the ecosystem for survival. This paper, presents a study on the application of SOS with Simulated Annealing (SA) to solve the well-known traveling salesman problems (TSPs). The TSP is known to be NP-hard, which consist of a set of $(n-1) ! / 2$ feasible solutions. The intent of the proposed hybrid method is to evaluate the convergence behaviour and scalability of the symbiotic organism's search with simulated annealing to solve both small and large-scale travelling salesman problems. The implementation of the SA based SOS (SOS-SA) algorithm was done in the MATLAB environment. To inspect the performance of the proposed hybrid optimization method, experiments on the solution convergence, average execution time, and percentage deviations of both the best and average solutions to the best known solution were conducted. Similarly, in order to obtain unbiased and comprehensive comparisons, descriptive statistics such as mean, standard deviation, minimum, maximum and range were used to describe each of the algorithms, in the analysis section. The oneway ANOVA and Kruskal-Wallis test were further used to compare the significant difference in performance between SOS-SA and the other selected state-of-the-art algorithms. The performances of SOS-SA and SOS are evaluated on different sets of TSP benchmarks obtained from TSPLIB (a library containing samples of TSP instances). The empirical analysis' results show that the quality of the final results as well as the convergence rate of the new algorithm in some cases produced even more superior solutions than the best known TSP benchmarked results.
\end{abstract}

Keywords: Symbiotic organisms search (SOS); simulated annealing (SA); traveling salesman problem (TSP); simulated annealing based symbiotic organisms search (SOS-SA).

\section{Introduction}

The traveling salesman problem (TSP) is an NP-hard problem, which has remained an interesting problem for a long time in the field of discrete or combinatorial optimization techniques, which are based on linear and nonlinear programming. The TSP presents the task of finding an optimum path through a set of given locations (cities), such that each location is passed through only once, and the salesman returns to the start location (Durbin, 1987; Durbin et al., 1989). In operational research, TSPs still remain one of the most challenging problems, which cannot be solved easily by using traditional optimization techniques such as enumeration methods and mathematical programming (Çunkaş and Özsağlam, 2009). Solving TSP optimally takes huge computational time and therefore the need for the development of fast heuristics that gives near optimal solution in a reasonable computational effort (Matai et al., 2010). While on small graphs the execution time may not be significant, on large datasets containing millions of vertices and edges the limitations (e.g., traceroutes, social graphs) of existing approaches become obvious. With the emergence of the Big Data era when we deal with huge graphs with different properties (e.g., sparse, power law, dense) there is a crucial need to develop novel techniques based on new paradigms and scalable algorithms. Among possible approaches are those inspired from metaheuristics which allow for a better exploration of the solution space and faster convergence to suboptimal solutions.

In the past decades, many metaheuristic based algorithmic strategies were proposed in the quest for finding near-optimum solutions to the TSPs, among which include Tabu Search (TS) (Knox, 1994), SA (Kirkpatrick et al., 1983), Genetic Algorithm (GA) (Johnson and McGeoch, 1997), Ant Colony Optimization (ACO) (Dorigo and Gambardella, 1997), Particle Swarm Optimization (PSO) (Shi et al., 2007), Artificial Immune System (AIS) (Farmer et al., 1986), Artificial Neural Network (ANN) (Jolai and Ghanbari, 2010), Elastic Net (EN) (Durbin et al., 1989), SOS (Cheng and Prayogo, 2014).

\footnotetext{
* Corresponding author:

Email: EzugwuA@ukzn.ac.za (A. E. Ezugwu)
} 
In this paper we focus on the SOS algorithm for reasons explained next. The algorithm draws inspiration from nature through the symbiotic relationships strategies, which exist among organisms in the ecosystem. The SOS algorithm was initially proposed to solve continuous engineering optimization problems. Several results (Tran et al., 2016; Cheng et al., 2015; Aulady, 2013; Verma et al., 2015), which have used the SOS algorithm as an optimization tool to find global optimum solutions, indicate that the algorithm shows a considerable robustness in its performance when tested on complex mathematical benchmark problems. Therefore, the potential of SOS in finding global solution to the aforementioned optimization problems makes it attractive for further investigation. Furthermore, since SOS has not gained wide recognition in solving discrete problems, such as, routing and assignment problems, we believe that demonstrating its effectiveness in solving TSP could pave the way for wide scale applicability in solving complex discrete problems.

The TSP optimization problem is considered to be a large-scale optimization problem, which makes it difficult to obtain satisfactory results by just using classical metaheuristic optimization algorithms such as SA, TS, GA and ACO. Recent researches have shifted focus to employing different hybridization techniques to solve all kinds of complex large scale optimization problems. The essence of the hybridization process is mainly to utilize the complimentary advantages and value-added information found in several algorithms and insufficient in single algorithm based approaches to enhance the efficiency of solving the large-scale problem like the TSP. Two recent researches on the application of SOS to solve related discrete optimization problems for instance, have shown that the classical version of the SOS algorithm still required some level of improvement for it to achieve better solution quality, as evident in the work presented in (Yu et al., 2016; Eki et al., 2015). The implementation results from these researches, shows that by combining basic SOS with some other algorithms, like the local search methods or solution representation, this significantly improves the computation efficiency and quality of the solutions. Another impact of the hybrid features is that it allows the SOS algorithm to easily escape from falling into local optimum.

While the specific objective of this paper is to show that the hybrid SOS is a promising candidate optimization solution for the TSP, the result also emphasizes the future applicability of the SOS algorithm augmented with SA in efficiently solving a wider range of complex discrete problems. The proposed SOS-SA method was implemented in Matlab and tested using TSP data sets (http://elib.zib.de/pub/mp-testdata/tsp/tsplib/tsp/) against other state-of-the-art algorithms such as Genetic Algorithm Particle Swarm Optimization Ant Colony Optimization (GA-PSO-ACO) (Deng et al., 2012), Adaptive Simulated Annealing Algorithm with Greedy Search (ASA-GS) (Geng et al., 2011), Multi-agent Simulated Annealing Algorithm with Instance-Based Sampling (MSA-IBS) (Wang et al., 2015), List-Based Simulated Annealing (LBSA) (Zhan et al., 2016), and Improved Discrete Bat algorithm (IBA) (Osaba et al., 2016). These sets of algorithms have been selected because of their common similarities in implementation techniques with the SOS-SA algorithm. Results show that the hybridized SOS-SA algorithm is able to achieve better results in solving most of the TSP benchmark problems with graphs ranging from 42 up to 33,810 cities.

The technical contributions of this paper are as follows:

i. Proposal of a new TSP optimization method, called simulated annealing based symbiotic organisms search optimization algorithm.

ii. Implementation of the proposed method using different scale of TSP benchmark instances.

iii. Performance comparison of the proposed hybrid method with other state-of-the-art algorithms (GAPSO-ACO, ASA-GS, MSA-IBS, LBSA, and IBA).

iv. Descriptive statistical validation of the SOS-SA results against other selected methods using different statistical analysis tests.

The remainder of this paper is organised as follows: Section 2 presents the related work; Section 3 provides a short description of the TSP problem; Section 4 presents the proposed SOS-SA method of solving TSP; while Section 5 describes and discusses the simulation results carried out on some benchmarked TSP instances; finally, conclusions and directions for future research are given in Section 6.

\section{Related works}

The TSP being a hard combinatorial optimization problem with high social interest, has over the past decades drawn the attention of the scientific communities, with a record number of optimization algorithms being proposed to address the minimization problem (Chen and Chien, 2011;). Interested readers may refer to (Wang et al., 2016; Kanda et al., 2016; Sundar and Rathinam, 2016; Cornu et al., 2016; Zhang and Zhou, 2016; Delgadillo et al., 2016; Zhang et al., 2016; Barbato et al., 2016; Mohan et al., 2017) for more recent results on the TSP cases. In this section we present some of the most representative results. 
The TSP being an NP-hard problem, which in most cases does not admit any constant factor approximation (Garey and Johnson, 1979, except in some exceptional cases: Bender and Chekuri, 2000 and Mohan et al., 2017), has resulted in the proposition of different optimization approaches, which are intended to provide solutions to the complex problem. In (Matai et al., 2010) for instance, two approaches of solving TSP were identified. The first approach uses the exact methods, of which guaranty of achieving optimal solution is greatly disadvantaged by the exponential cost of execution time that scale with the problem dimension. Thus, this approach is considered unsuitable for solving large TSPs. Two common examples of this approach are the dynamic programming (Bellman, 1962), and branch and bound (Lawler and Wood, 1966; Volgenant and Jonker, 1982). The second approach is known as the approximation algorithm. This approach only gives near optimal solution, but does not guarantee optimal solution. One main advantage of this approach is that it requires minimal computational effort regardless of the problem dimension. The approximation algorithms can further be classified into local search and heuristic optimization algorithms. The local search or improvement heuristics are usually applied to improve the quality of TSP solution generated. Examples of these heuristics are the 2-Opt (Johnson, 1990), and 3-Opt (Lourenço et al., 2003) exchange heuristics.

In recent years, most of the new proposed methods for solving TSP indicate shift towards improving the solution quality of the traditional based heuristics, through the development of hybrid algorithms that overcome the disadvantages of the individual algorithms. Recent studies also show that the combined efforts of two or more algorithms are usually more effective than the effort of each individual algorithm (Lin et al., 2016; Tsai et al., 2004; Katayama et al., 2000; Talbi, 2002). This generally implies that often at times the capabilities of most hybrid algorithms are more effective and efficient than that of the individual algorithms.

Some of the existing hybrid heuristic optimization based approaches used for searching near optimal solution for TSPS outside those aforementioned in the previous section include: a hybrid of genetic algorithm particle swarm optimization ant colony optimization (GA-PSO-ACO) (Deng et al., 2012), adaptive simulated annealing algorithm with greedy search (ASA-GA) (Geng et al., 2011), multi-agent simulated annealing algorithm with instance-based sampling (MSA-IBS) (Wang et al., 2015), list-based simulated annealing (LBSA) (Zhan et al., 2016), invasive weed colony optimization (IWO) (Zhou et al., 2015), mosquito host-seeking algorithm (MHSA) (Feng et al., 2009), an improved discrete bat (IBA) algorithm (Osaba et al., 2016), Discrete Cuckoo Search (DCS) algorithm (Ouaarab et al., 2014), genetic simulated annealing ant colony system with particle swarm optimization (Chen and Chien, 2011) and the symbiotic organisms search (SOS) algorithm (Yu et al., 2016; Eki et al., 2015). These algorithms are problem independent and have strong global search capability, while the hybrid features allows them to easily escape from falling into local optimum. Subsequently, brief reviews of the related literature are discussed.

GA-PSO-ACO (Deng et al., 2012) is an algorithm which combines the evolution ideas of the genetic algorithm, particle swarm optimization and ant colony optimization algorithm to solve the travelling salesman problem. The implementation entails applying the combination of randomicity, rapidity and wholeness of the genetic algorithm and particle swarm optimization methods to achieve a series of sub-optimal solutions. The resulting solution is later exploited by the ant colony optimization procedure, by taking the advantage of the parallel, positive feedback and high accuracy of solution to implement solving of whole problem. Osaba et al. (2016) proposed an improved discrete version of bat algorithm (IBA) for solving both symmetric and asymmetric TSP. The algorithm which was tested on 37 TSP instances produced an interesting result, which outperformed the other alternative benchmarked algorithms in most of the cases.

Geng et al. (2011) proposed an adaptive hybrid algorithm that combines the problem solving efforts of simulated annealing and greedy search technique (ASA-GS) to solve the TSP. The greed search technique assist in speeding up the solution convergence rate, while the hybrid algorithm achieves better trade-off between computation time and solution quality. The algorithm evaluation shows that it has good scalability and performs better even with large-scale TSP instance. A combination of multi-agent and simulated annealing with instance based sampling (MSA-IBS) was proposed by Wang et al. (2015) and used to solve the TSP. The hybrid process exploited the learning ability of the instance-based search algorithm to improve the sampling efficiency of the simulated annealing, the algorithm competed favourably in terms of solution quality and utilization of system resource (like cpu time) as compared to the ASA-GS algorithm. In the work of Zhan et al. (2016), a list-based simulated annealing algorithm was proposed also to solve the TSP. The algorithm uses the effectiveness and parameter sensitivity of the list-based cooling schedule to control temperature reduction in SA, which is used as acceptance criteria for choosing candidate solution. The simulation result of the LBSA shows that it is robust and performs fairly well compared to some other state-of-the-art algorithms.

Similar works that uses simulated annealing can be found in (Chen and Chien, 2011), where the authors proposed a new hybrid optimization method for solving the TSP. This paper consists of a hybrid of genetic simulated annealing, ant colony system, and particle swarm optimization technique. In the implementation, the 
ant colony system is used to generate the initial solution for the genetic algorithm's procedure, after which the initial solution is fine-tuned with the simulated annealing, which generates better solutions than the previous one. The role of the particle swarm optimization technique is to facilitate the exchange of pheromone information among the populations in the ant colony system after a predefined number of cycles. The simulation results showed that the hybrid algorithm performed better compared to the other algorithms. In (Malek et al., 1998) parallel and serial version of simulated annealing and tabu search algorithms was implemented and used to solve the TSP. In (Fang et al., 2007), particle swarm optimization with simulated annealing was implemented to solve TSP. The simulated annealing was applied to slowdown the degeneration of PSO swarm and to also increase the swarm's diversity. In addition, the choice of selecting the benchmarked algorithms that were compared with the proposed SOS-SA was made considering two significant characteristics; (i) population based algorithm implementations namely, GA-PSO-ACO, IBA and SOS, (ii) SA-based hybrid algorithm implementations namely, ASA-GS, MSA-IBS and LBSA.

Due to the wider interest of this area of study, summarising all the related materials available in the literatures can be a daunting task to embark on. Therefore, the interested readers are referred to the following materials for further information on TSP and its computational solution (Applegate et al., 2011, Reinelt, 1998; Jünger et al., 1995).

In this work we will show that existing algorithms such as GA-PSO-ACO, MSA-IBS, LBSA, SOS, and IBA underperform our hybrid algorithm SOS-SA which enables SOS to escape local minimums and improves in some cases some of the best results known so far.

\section{Problem Formulation for TSP}

The TSP is a well-known combinatorial optimization problem that has for the past decades attracted the interest of research communities. There are different solution approaches proposed in the literatures, which are currently being used to solve the three classes of the TSPs namely, the symmetric, asymmetric and multi traveling salesman problems. The TSP problems are said to be NP-hard optimization problems, which mean that there is no known polynomial time algorithm that can specifically guarantee the attainment of its optimal solution and that is why heuristic or approximation approaches remain the preferred methods often recommended for solving the TSP problems. The TSP has numerous application areas which were highlighted in (Matai et al., 2010), some of which include: drilling of printed circuit boards, overhauling gas turbine engines, $x$-ray crystallography, computer wiring, crew scheduling, interview scheduling, mission planning, vehicle routing, mask plotting in PCB production, and design of global navigation satellite system surveying networks. In this paper, of interest is the symmetric travelling salesman problem.

The symmetric TSP can also be defined in terms of a complete undirected graph $G=(V, E)$, where the set $V=$ $\{1,2, \ldots, n\}$ is the vertex set, $E=\{(i, j): i, j \in V, i<j\}$ is an edge set (Matai, Singh, and Mittal, 2010). A cost matrix $X=\left(x_{i, j}\right)_{n \times n}$ is defined on $E$. The cost matrix satisfies the triangle inequality whenever $x_{i, k}+x_{j, l} \leq$ $x_{i, l}+x_{j, k}$ for all $1 \leq i<j \leq n, 1 \leq k<l \leq n$, or $x_{i, j} \leq x_{i, k}+x_{k, j}$, for all $i, j, k$. In particular, this is the case of planer problems for which the vertices are points $d_{i}=\left(q_{i}, p_{i}\right)$ in the plane, and $x_{i, j}=$ $\sqrt{\left(q_{i}-q_{j}\right)^{2}+\left(p_{i}-p_{j}\right)^{2}}$ is the Euclidean distance. The triangle inequality is also satisfied if $x_{i j}$ is the length of a shortest path from $i$ to $j$ on $G$. Also, in the classical problem in combinatorial optimization (Ozcan, and Erenturk, 2004), the TSP can be defined as follows: given $n$ cities and the distance $x_{i j}$ between them, the shortest distance $\varphi$ through all the cities can be computed by minimizing the function expressed in Eq. 1 .

$$
f(\varphi)=\sum_{i=1}^{n} x_{\varphi(i), \varphi(i+1)}+x_{\varphi(n), \varphi(1)}
$$

where, $\varphi$ a set of permutations $\varphi \rightarrow\{1,2, \ldots, n\}$ with $n$ being all the possible number of tours of the problem, and $f(\varphi)$ representing the cost of the permutation $\varphi$.

\section{Simulated Annealing based Symbiotic Organisms Search (SOS-SA)}

In this section, the two basic search algorithms that make up the hybrid algorithm proposed for solving the TSP problem are discussed. 


\subsection{Symbiotic Organisms Search Algorithm}

In the real world, the close association between two or more different organisms of different species living together in an ecosystem, often but not necessarily benefits each member. When the relationship is beneficial to both organisms, it is called mutualism and symbiosis. When it is beneficial to one without effect on the other it is called commensalism, and when it is beneficial to one and detrimental to the other it is called parasitism. Almost all the metaheuristics optimization algorithms are bio-inspired from natural biological phenomena, which follow in the same trend with the symbiotic relationship explained in this section. The SOS algorithm which applies the same symbiotic relationship principles seen among organisms in nature in solving optimization problems differs greatly from other similar metaheuristic algorithms, in the sense that it does not require any algorithm-specific parameters (Cheng et al., 2015). One major advantage of this is that an improper tuning related to algorithm-specific parameters would lead to an increase in computational time and premature convergence.

The algorithm is implemented by first creating a random ecosystem or population matrix, with each row (known as organism) representing a candidate solution to the corresponding problem. The size of the population often referred to as the ecosystem size (eco_size) defines the number of organisms that make up the ecosystem, a parameter usually set by the user. The search process starts immediately after the initial ecosystem has been created and it comprises of continuous interactions among the ecosystem member organisms. The interactions follow the three phases of symbioses interaction namely, mutualism, commensalism, and parasitism, which the organisms adopt to increase their survival and fitness advantage for a prolonged period of time. In the course of the interaction process, an organism would either receive a benefit or harmed, in which case the one that benefits evolve to a fitter organism whereas the one that is harmed is eliminated. Iteratively the best organism is modified and updated until the stopping criterion is reached. The SOS is implemented using the pseudocode shown in algorithm listing 1.

The classical SOS algorithm was designed to operate on real-value variables, and this would probably limits it application to discrete optimization problems, a conversion function, which converts the variables from real values to integer values is given in equation 4 . This idea follows similar concept proposed in (Tran et al., 2016) to make SOS suitable for application to solve the TSP. Consider a distance or cost matrix where $x_{i, j}$ is the distance of $i^{\text {th }}$ city to $j^{\text {th }}$ city, which is optimized by the SOS algorithm during the search process. However, the ecosystem population is created before the start of the search process and the population consist of all the feasible solutions or possible associated tour costs defined by the distance matrix expressed in Eq. 2 .

$$
X_{m \times n}=\left[\begin{array}{cccc}
x_{1,1} & x_{1,1} & \cdots & x_{1, n} \\
x_{2,1} & x_{2,2} & \cdots & x_{2, n} \\
\vdots & \vdots & \vdots & \vdots \\
x_{i, 1} & x_{i, 2} & x_{i, j} & x_{i, n} \\
\vdots & \vdots & \ddots & \vdots \\
x_{m, 1} & x_{m, 2} & \cdots & x_{m, n}
\end{array}\right]
$$

where $m$ represent the ecosystem size or the problem size and $n$ represent the number of elements in a vector of decision variables in the problem under consideration. The decision variables for the TSP, which consists of the cities and their associated costs are represented as a vector, which is expressed in Eq. 3.

$$
X=\left[x_{i, 1}, x_{i, 2}, \ldots, x_{i, j}, \ldots, x_{i, n},\right]
$$

Therefore, to convert the real-value variables to integer values, the function expressed in Eq. 4 is implemented.

$$
X_{i, j}=\operatorname{round}\left\{x_{i, n} \times \operatorname{swap}(\varphi, i+1, j)\right\}
$$

where $x_{i, n}$ is the swapped state value, that is, the value for a particular tour through the set of given cities or points. Usually, a neighbour state is obtained by randomly swapping the order of two cities. The swap function represents the total number of swap action for each tour, while $n$ represents the number of tours. The function rounds in Matlab is used to round each point of $X_{i, j}$ to the nearest integer less than or equal to that point.

The SOS optimization strategy is performed by following three search and update phases (i.e., mutualism, commensalism, and parasitism) as presented subsequently.

Mutualism phase: In the mutualism phase, two organisms $X_{i}$ and $X_{j} \mid i \neq j\left(X_{j}\right.$ is selected randomly from the population) are considered on the bases of mutual interest. The association between $X_{i}$ and $X_{j}$ is to increase 
mutual survival of the two organisms in the ecosystem. The resulting solution $X_{i}^{\prime}$ and $X_{j}^{\prime}$ are computed as shown in Eqs.5 and 6:

$$
\begin{aligned}
& X_{i}^{\prime}=X_{i}+\operatorname{rand}(0,1) \times\left(X_{\text {best }}-\text { Mutual }_{\text {vect }} \times K_{1}\right) \\
& X_{j}^{\prime}=X_{j}+\operatorname{rand}(0,1) \times\left(X_{\text {best }}-\text { Mutual }_{\text {vect }} \times K_{2}\right)
\end{aligned}
$$

The mutual vector denoted by Mutual $_{\text {vect }}$ is expressed as shown in Eq. 7.

$$
\text { Mutual }_{\text {vect }}=\frac{X_{i}+X_{j}}{2}
$$

The rand $(0,1)$ function is a vector of uniformly distributed random numbers between 0 and 1 . The values of the benefit factors $K_{1}$ and $K_{2}$ are determined randomly as either 1 or 2 , and represents the level of benefit to each of the two organisms $X_{i}$ and $X_{j}$ (where 1 and 2 denotes adequate and huge benefit that can be received by both $X_{i}$ and $X_{j}$ in their current mutual symbiotic states). The organism with the best objective or fitness function value in terms of the degree of adaptation in the ecosystem is represented by $X_{\text {best }}$. The Mutual $_{\text {vect }}$, signifies mutualistic characteristics exhibited between the two organism to increase their survival advantage. It should be noted that any update for any one of the two organisms is computed only if its new fitness function value denoted by $f\left(X_{i}^{\prime}\right)$ or $f\left(X_{j}^{\prime}\right)$ is better than the previous solutions, $f\left(X_{i}\right)$ and $f\left(X_{j}\right)$. Given the above Eqs. 5 and 6 become:

$$
\begin{aligned}
& X_{i}^{\prime}=X_{i}+\operatorname{rand}(0,1) \times\left(X_{\text {best }}-\text { Mutual }_{\text {vect }} \times K_{1}\right), \text { if } f\left(X_{i}^{\prime}\right)>f\left(X_{i}\right) \\
& X_{j}^{\prime}=X_{j}+\operatorname{rand}(0,1) \times\left(X_{\text {best }}-\text { Mutual }_{\text {vect }} \times K_{1}\right), \text { if } f\left(X_{j}^{\prime}\right)>f\left(X_{j}\right)
\end{aligned}
$$

Commensalism phase: In this phase, the organism $X_{i}$ selected randomly from the ecosystem strives to increase its benefits from its association with $X_{j}$. This kind of symbiotic association only places $X_{i}$ at an advantage position, over $X_{j}$, even though, $X_{j}$ is not harmed in the process. The new solution emanating from the symbiotic relationship is calculated as shown in $E q$. 9:

$$
X_{i}^{\prime}=X_{i}+\operatorname{rand}(-1,1) \times\left(X_{\text {best }}-X_{j}\right) \text { if } f\left(X_{i}^{\prime}\right)>f\left(X_{i}\right)
$$

Parasitism phase: Also in Cheng and Prayogo (2014), an example of parasitic symbiotic relationship was illustrated by using the association that exists among three organisms, the plasmodium parasite, anopheles mosquito and the human host. In this kind of association, the human host is harmed, the anopheles mosquito, which is the parasite carrier, is left unharmed, while the plasmodium parasite thrives and reproduces inside the human body. In the SOS model, by mimicking the aforementioned parasitic symbiotic behaviours, $X_{i}$ is assigned a role akin to the anopheles mosquito through the creation of an artificial vector (or parasite vector) $P_{v e c}$ in the search space, by fine-tuning the randomly selected dimension of organism $X_{i}$. Then, the organism $X_{j}$ is selected randomly from the ecosystem and serve as host to $P_{v e c}$. Then, $P_{v e c}$ will try to replace $X_{j}$ in the ecosystem. If $P_{v e c}$ has a better fitness value than $X_{j}$, then $X_{j}$ is replaced by $P_{v e c}$, otherwise, $X_{j}$ develops an immunity from $P_{v e c}$, which will invariably cease to exist in the ecosystem. The procedure for the classical SOS algorithm proposed by Cheng and Prayogo (2014) is presented in the algorithm listing 1 below.

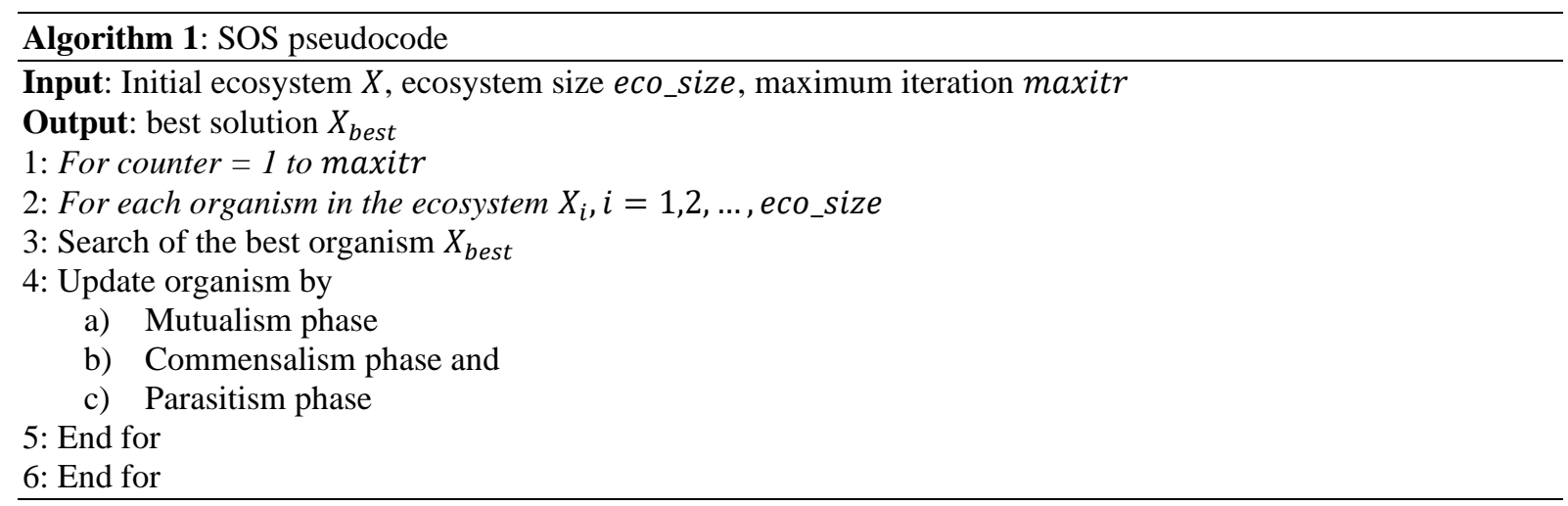

The SOS algorithm though efficient in solving complex optimization and discrete engineering problems, still has high probability of plunging into local optimum (Vincent, et al., 2016). Therefore, the SOS-SA algorithm has been proposed to overcome this shortcoming. 


\subsection{Simulated Annealing Algorithm}

The application of SA to solve TSP was first introduced by Kirkpatrick et al. (1983). The process begins by considering a solution space $S$ of a particular tour through the set of given cities or points $X_{i} \mid i=1,2, \ldots, n$, with an update solutions $X_{i}^{\prime}$ created by randomly switching the orders of two cities. The energy function or fitness function, which represents the length of route $X_{i}$, is denoted by $f\left(X_{i}\right)$. The relative change in cost $\Delta f$ between $X_{i}$ and $X_{i}^{\prime}$ is expressed as $\Delta f=\frac{f\left(X_{i}^{\prime}\right)-f\left(X_{i}\right)}{f\left(X_{i}\right)}$. Beginning with the initial solution, only the solution which results in smaller energy value than the previous solution is accepted by the algorithm, in other words, a solution is only accepted when the fitness value of $f\left(X_{i}^{\prime}\right)<f\left(X_{i}\right)$. However, accepting or rejecting a new solution with higher fitness values for $X^{\prime}$ can be based on the acceptance probability function given as follows (Eq. 10):

$$
P\left(\Delta f, T_{k}\right)=\left\{\begin{array}{ll}
e^{\left(\frac{-\Delta f}{T_{k}}\right)}, & \Delta f>0 \\
1, & \Delta f \leq 0
\end{array} \quad \text { for } T_{k}>0\right.
$$

where $T_{k}$ is the parameter temperature at the $k^{\text {th }}$ instance of accepting a new solution route, and for any given $T$, for $\Delta f>0, P$ is greater for smaller values of $\Delta f$, which means that for the new solution $X_{i}^{\prime}$ that is only slightly more costly than the current solution $X_{i}$ is more likely to be accepted than the new solution $X_{i}^{\prime}$ that is much more costly than the current solution $X_{i}$. The value of $T$, which is an important control parameter, decreases proportionally with $P$, that is as the $\lim _{T \rightarrow 0^{+}} e^{\left(\frac{-\Delta f}{T_{k}}\right)}=0, \Delta f>0$. Therefore, as the value of $T$ decreases, the probability of accepting a degraded route also decreases. In this paper the following cooling schedule is adopted (Eq. 11):

$$
T_{k+1}=\alpha T_{k}
$$

Where, $\alpha$ denotes the cooling coefficient, which is some random constant values between 0 and 1 , it is also the rate at which the temperature is lowered each time a new solution $X_{i}^{\prime}$ is discovered. The SA procedure is as presented in the algorithm listing 2 below:

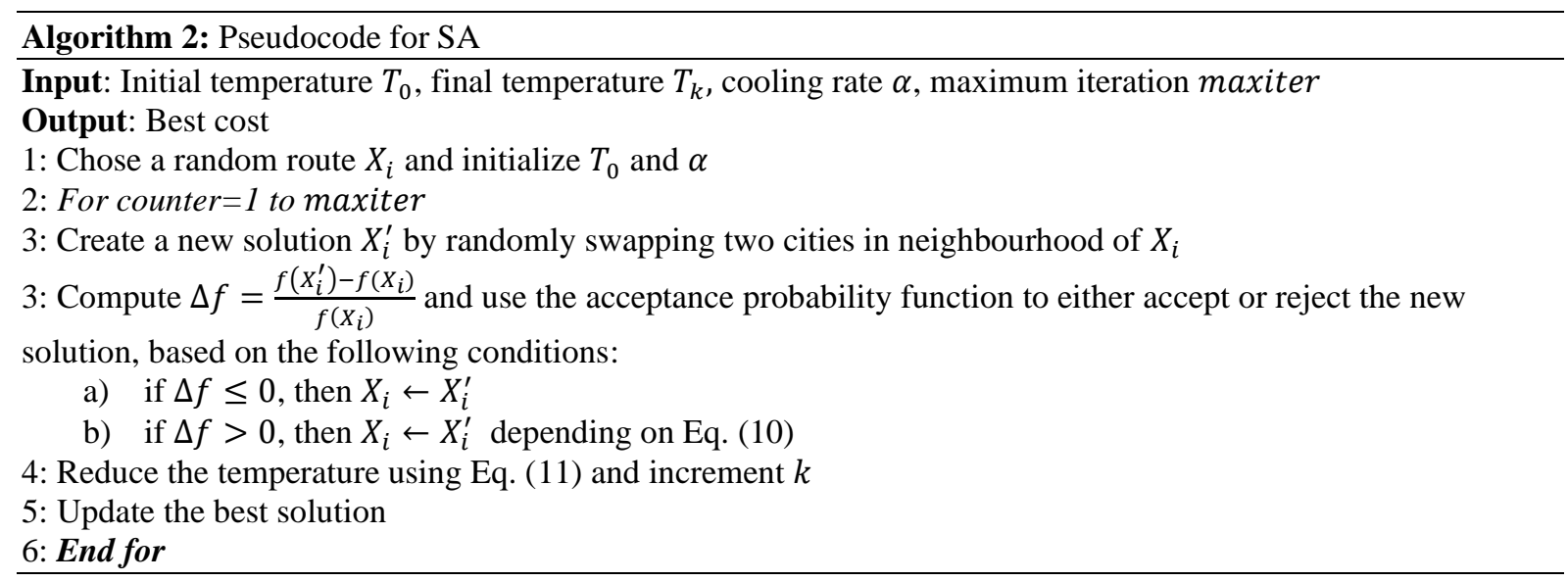

\subsection{SOS-SA Framework for Solving TSP}

The SOS-SA algorithm is a hybrid of symbiotic organisms search and simulated annealing algorithm. The SA is a local search metaheuristic algorithm widely used for solving both discrete and continuous optimization problems (Kirkpatrick et al., 1983). One of the main benefits of SA lies in its ability to escape the problem of getting stuck in a local minimum by allowing hill-climbing moves to search for a global solution. Therefore, a hybrid approach is proposed by introducing SA is to assist the SOS in avoiding being trapped into local minimum and to also increase its level of diversity while searching for optimum solution in the problem search space. Exploiting the fast optimal search capability of the SOS algorithm with the hill-climbing probability jump property of the SA, as described in algorithm listing 1 and 2 above, a new hybrid algorithm (SOS-SA) is proposed to solve the TSP problem. The steps of the hybrid SOS-SA algorithm are then described in algorithm listing 3 . 


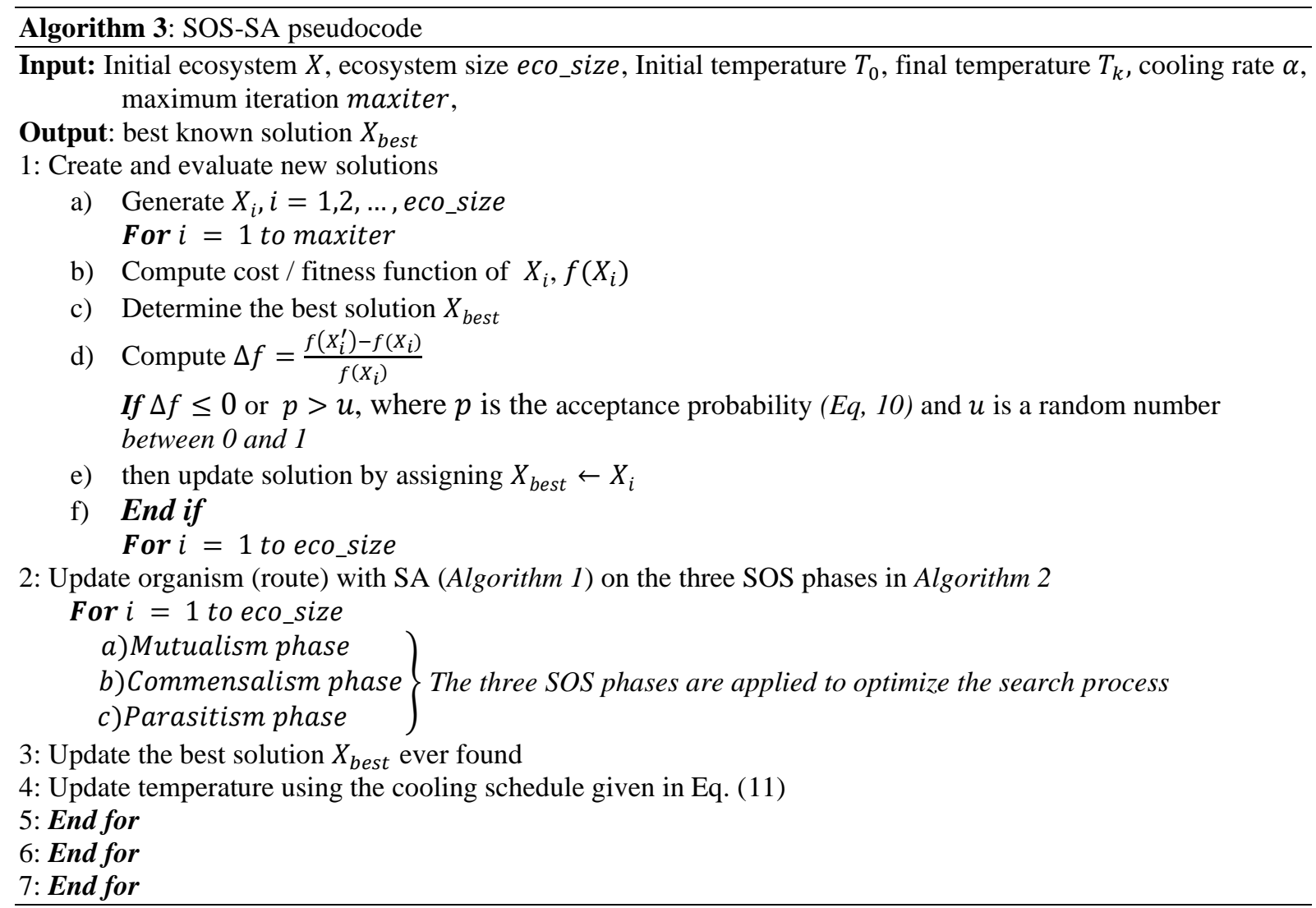

The SOS-SA algorithm follows through all the steps highlighted in algorithm 3, starting by first initializing the ecosystem $X_{i}$ of size $e c o \_s i z e$. Then creating and evaluating each new organism's positions by computing and comparing their respective tour cost functions, such that the organism with the least tour cost is selected as $X_{b e s t}$ . Iteratively, the process is repeated by updating the current solution with the best solution ever found until the organism with the global best solution is discovered. The SOS-SA algorithm uses the three SOS relationships' phases to update the organism. The algorithm finishes when maximum iterations criterion is attained. Otherwise, the algorithm continues to calculate new positions. However, stopping condition is quite an important factor that can determine the final result of the simulation. For example, if the algorithm is stopped too early, the approximation of the solution might not be even close to the targeted global optimum, and prolonging the simulation incurs unnecessary huge amount of computational effort and time. A fixed generation number of 1,000 was set as the stopping condition for the simulation and this setting was adequate, as it limits unproductive work. Fig. 1 illustrates the SOS-SA algorithm procedures. 


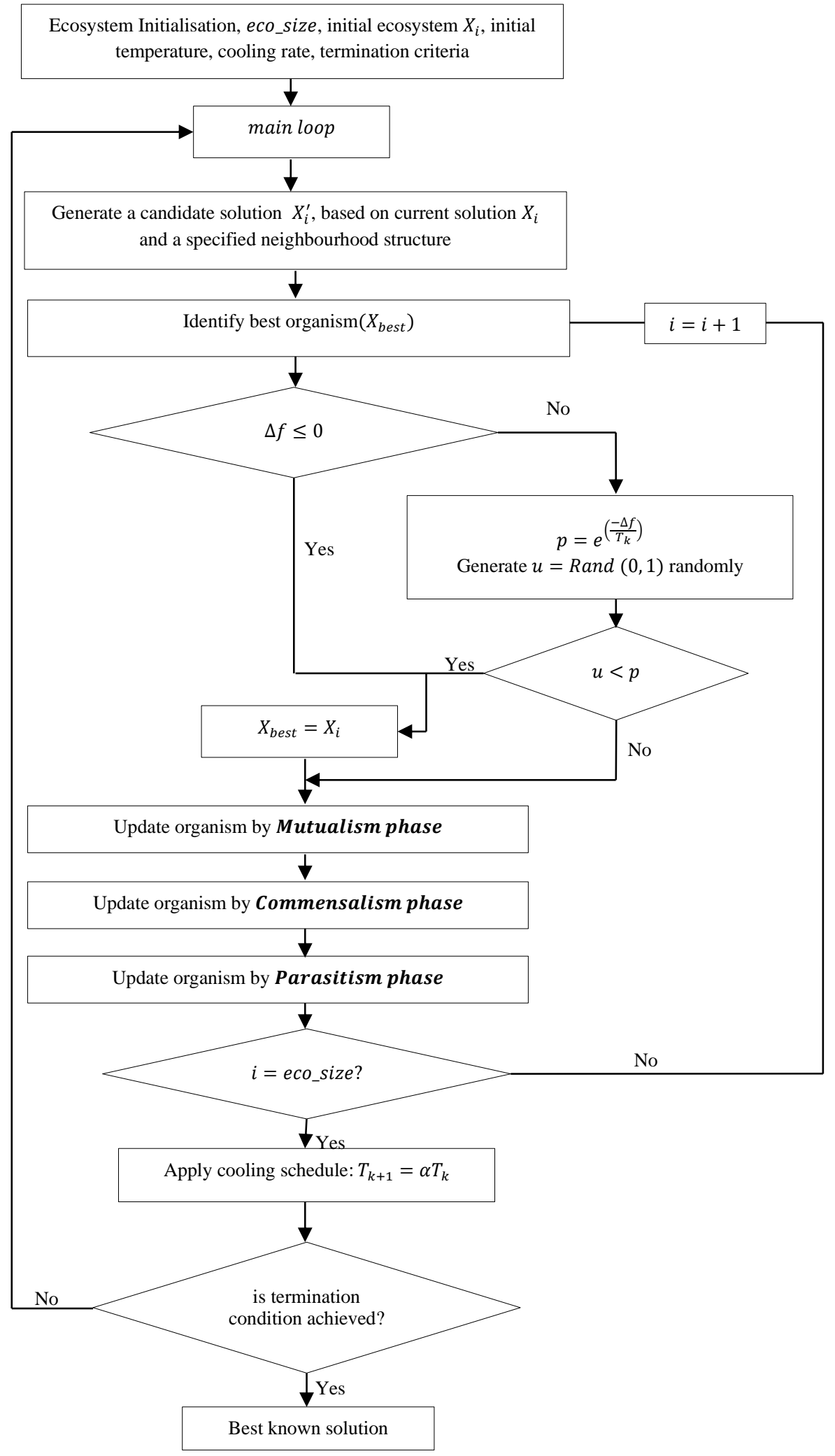

Fig.1. Flowchart for the SOS-SA algorithm 


\section{Simulation and Result}

The TSP experimental data sets used in this paper were obtained from the MP-TESTDATA, which covers: The TSPLIB Symmetric Traveling Salesman Problem Instances ${ }^{2}$ and Best known solutions for symmetric TSPs ${ }^{3}$. In order to evaluate the performance of the SOS-SA algorithm, two sets of simulation experiments were conducted.

The first experiment was carried out to evaluate the performance of the proposed SOS-SA algorithm, GA-PSOACO, MSA-IBS, and LBSA, against the best known solution from the TSPLIB, on six (6) benchmarked TSP results and their results as presented in Table 2.Similar comparisons were also carried out for SOS-SA, SOS, GA-PSO-ACO, MSA-IBS, and LBSA, to compare the quality of solutions for each algorithm. The comparison results are as presented in Tables 3, 4, 5, 6, 7, 8 and 9.

The second experiment was carried out to evaluate the computational effort of the SOS-SA algorithm based on execution time, on 40 benchmarked TSP instances and the results are presented in Table 10. In Table 2, the boldly highlighted column indicate those areas where the SOS-SA competed and performed favourably with the best known solution of the optimal TSPLIB instance and the other three state-of-the-art algorithms. Several statistical tests were also conducted to validate the obtained results, as depicted in Tables 11 (on 40 benchmarked TSP instance), 12 (on 20 benchmarked TSP instance), and 13 (on 16 benchmarked TSP instance). The average of 20 trials was taken and the number of the outer iteration times was set to 1,000 and 2,000 respectively.

\subsection{Experimental configurations}

The experimental results presented in this section demonstrate the scalability, effectiveness and efficiency of the SOS-SA in solving different TSP instances ranging from 42 up to 33,810 cities. The simulation time and number of iterations used to solve the TSP instances on a single machine are similarly presented here. The experimental testing platform for the SOS-SA and SOS algorithms were conducted on a $2.83 \mathrm{GHz}$ CPU Desktop with 2GB RAM, while the implementation software is Matlab R2014b. For the implementation of MSA-IBS and LBSA algorithms, the experiments were run on a $2.8 \mathrm{GHz}$ PC with 2GB RAM, ASA-GS was run on 2.83 GHz PC, and GA-PSO-ACO was run on Intel Core i52410 Laptop with $2.30 \mathrm{GHz}$ and 4GB RAM. For the entire TSP instance tested, the maximum iterations for the outer loop were set to 1,000 , which correspond to the iteration times set for the other compared algorithms. In the case of other algorithms, the selection population size is dependent on the scale of problem instance, which is the case with SOS-SA and SOS algorithms. As stated in (Zhan et al., 2016), the algorithm execution stopping condition is either when an optimal solution is found or when the iteration times of the outer loop reaches 1,000 .

Since parameter selection may significantly influence the solution's quality of each algorithm performance, the parameter settings for all the simulations conducted are presented in Table 1.

Table 1: Experimental parameter configuration

\begin{tabular}{ll}
\hline SOS-SA Parameters & SOS Parameter \\
\hline Population size $=50,100$ & Population size $=100$ \\
Maximum iteration $=1000,2000$ & Maximum iteration= 2000 \\
Initial temperature $=0.025$ & Initial temperature $=0.025$ \\
Cooling rate $=0.99$ & Cooling rate $=$ NA \\
Number of cities to swap $=2$ & Number of cities to swap $=$ NA
\end{tabular}

LBSA Parameters

Population size $=100$

Maximum iteration $=1000$

Inertia temperature $=$ produced according to initial acceptance probability $\boldsymbol{p}_{\mathbf{0}}$ in the range of $\mathbf{1 0}^{-\mathbf{2 0}}$ to 0.9

Cooling rate is adaptively selected as follows: $\boldsymbol{t}_{\boldsymbol{i}}=\frac{-\boldsymbol{d}_{\boldsymbol{i}}}{\ln \left(\boldsymbol{r}_{\boldsymbol{i}}\right)}$, where $\boldsymbol{r}$ is a random number and $\boldsymbol{d}$ is the difference of objective function values.

Number of cities to swap $=2$

\begin{tabular}{ll}
\hline ASA-GS Parameter & MSA-IBS \\
\hline Population size $=$ NA & Population size $=$ NA \\
Maximum iteration $=1000$ & Maximum iteration= NA \\
Initial temperature $=1000$ & Initial temperature $=$ NA \\
Cooling rate $=\left(\left(\boldsymbol{\alpha} \times \boldsymbol{N}^{\wedge} \mathbf{0 . 5}-\mathbf{1}\right)\right) /\left(\boldsymbol{\alpha} \times \boldsymbol{N}^{\wedge} \mathbf{0 . 5}\right), \mathrm{N}=$ no. of cities $\boldsymbol{\alpha}=\mathbf{1}$ & Cooling rate $=$ NA \\
Number of cities to swap $=$ NA & Number of cities to swap = NA \\
\hline IBA & GA-PSO-ACO \\
\hline
\end{tabular}

\footnotetext{
${ }^{2}$ http://elib.zib.de/pub/mp-testdata/tsp/tsplib/tsp/

${ }^{3}$ http://elib.zib.de/pub/mp-testdata/tsp/tsplib/stsp-sol.html
} 


\begin{tabular}{ll}
\hline Population size $=50$ & Population size $=100$ \\
Maximum iteration $=1000$ & Maximum iteration $=1000$ \\
Initial temperature $=$ NA & Initial temperature $=$ NA \\
Cooling rate $=$ NA & Cooling rate $=$ NA \\
Number of cities to swap $=$ 2-opt \& 3-opt & Number of cities to swap = NA
\end{tabular}

\subsection{Evaluation}

Table 2, describes the summary of the best known results so far obtained using the SOS-SA algorithm. Where the second column represents the name of the TSP instance, the third column represents the best known solution length taken from the TSPLIB, the fourth column represents the length of the best known solution found by SOS-SA algorithm, the fifth column represents the average length of the solution found by SOS-SA algorithm, and the sixth column represents the percentage deviation of the SOS-SA best solution. The SOS-SA best solution percentage deviation (PDbest), which determines the closeness of the solution to the best known solution (BKS), is calculated as shown in Eq. 12:

$$
\text { PDbest }=\frac{(\text { Best }-B K S)}{B K S} \times 100
$$

where Best denotes the best length value for each algorithm for the total number of runs under each problem instance.

The percentage deviation of the SOS-SA mean solution was also computed and used to compare it performance with the best known solution and other algorithms. The percentage deviation of the mean solution is subsequently defined as follows:

$$
P D \text { mean }=\frac{(\text { Mean }-B K S)}{B K S} \times 100
$$

where Mean denotes the average length value for each algorithm for the total number of runs under each problem instance.

\subsection{Discussion of results}

Table 2 demonstrates the extreme performance capability of the SOS-SA algorithm in comparison with other state-of-the-art algorithms. The new algorithm outperformed all the three algorithms and this include the best known solution so far "BKS" in the six TSP instance examined with percentage deviation (PDbest) $<0$ and performance accuracy that is above $100 \%$. Though the SOS-SA share some common characteristics with GAPSO-ACO based on the SOS component, and also due to the fact that each algorithm has a strong global search capability, the GA-PSO-ACO still has very strong tendency of falling into a local minimum, for which the SA component in the SOS-SA is able to prevent. As can be seen from the results shown in Table 2, MSA-IBS and LBSA competed relatively and favourably with over $99 \%$ performance accuracy against the best known solution in all the instances. The possible challenge with the two algorithms can be traced to few factors, some of which include the use of several parameters, high computation and communication cost incurred during the iterative execution of the algorithm, especially for the multi-agent based MSA-IBS algorithm. Fig. 2 illustrates the performance evaluation of the SOS-SA algorithm among other three algorithms including the BKS based on computed percentage deviation. 
Table 2: Best-so-far solutions found by SOS-SA algorithm compared with the beset know solution from TSPLIB and other algorithms, the best results are highlighted in bold.

\begin{tabular}{|c|c|c|c|c|c|c|c|c|c|c|c|c|c|c|c|c|c|c|}
\hline \multirow[t]{2}{*}{$\mathrm{S} / \mathrm{N}$} & \multirow[t]{2}{*}{ Instance } & \multirow[t]{2}{*}{$\mathrm{BKS}^{\mathrm{a}}$} & \multicolumn{4}{|c|}{ GA-PSO-ACO [Deng et al., 2012] } & \multicolumn{4}{|c|}{ MSA-IBS [Wang et al., 2015] } & \multicolumn{4}{|c|}{ LBSA [Zhan et al., 2016] } & \multicolumn{4}{|c|}{ SOS-SA } \\
\hline & & & Best $^{\text {b }}$ & Mean & Diff. & PDbest $(\%)^{c}$ & Best & Mean & Diff. & PDbest $(\%)$ & Best & Mean & Diff. & PDbest (\%) & Best & Mean & Diff. & PDbest $(\%)$ \\
\hline 1 & dantzig42 & 699 & NA & & NA & NA & NA & & NA & NA & NA & & NA & NA & 679 & 699.4823 & -20 & -2.86 \\
\hline 2 & Berlin52 & 7542 & 7544.37 & 7544.37 & 2.37 & 0.03 & 7542 & 7542 & 0 & 0 & 7542 & 7542 & 0 & 0 & 7540 & 7541.107 & -2 & -0.03 \\
\hline 3 & Pr76 & 108159 & NA & NA & NA & NA & NA & NA & NA & NA & NA & NA & NA & NA & 107899 & 107899 & -260 & -0.24 \\
\hline 4 & Rat99 & 1211 & 1218 & 1275 & 7 & 0.578 & 1211 & 1211.04 & 0 & 0 & 1211 & 1211.1 & 0 & 0 & 1210 & 1210.108 & -1 & -0.08 \\
\hline 5 & Pr107 & 44303 & 44316 & 44589 & 13 & 0.029 & 44303 & 44379.88 & 0 & 0 & 44303 & 44392.25 & 0 & 0 & 44301 & 44302.83 & -2 & -0.01 \\
\hline 6 & Pr124 & 59030 & 59051 & 60157 & 21 & 0.036 & 59030 & 59032.88 & 0 & 0 & 59030 & 59031.8 & 0 & 0 & 58985 & 59010.65 & -45 & -0.08 \\
\hline
\end{tabular}

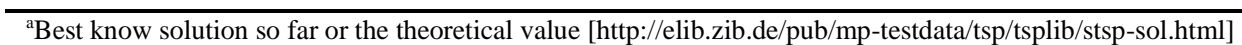

${ }^{\mathrm{b} B e s t}$ known solution for each of the algorithms

${ }^{\mathrm{c}}$ Relative percentage error for the results obtained by 10 runs. 
The negative signs against the difference (Diff.) values and percentage deviations (PDmean and PDbest) in Table 2 and other Tables are left as indication to show that the SOS-SA solution outperformed in some cases the best known solution, although percentage deviation is supposed to be an absolute value.

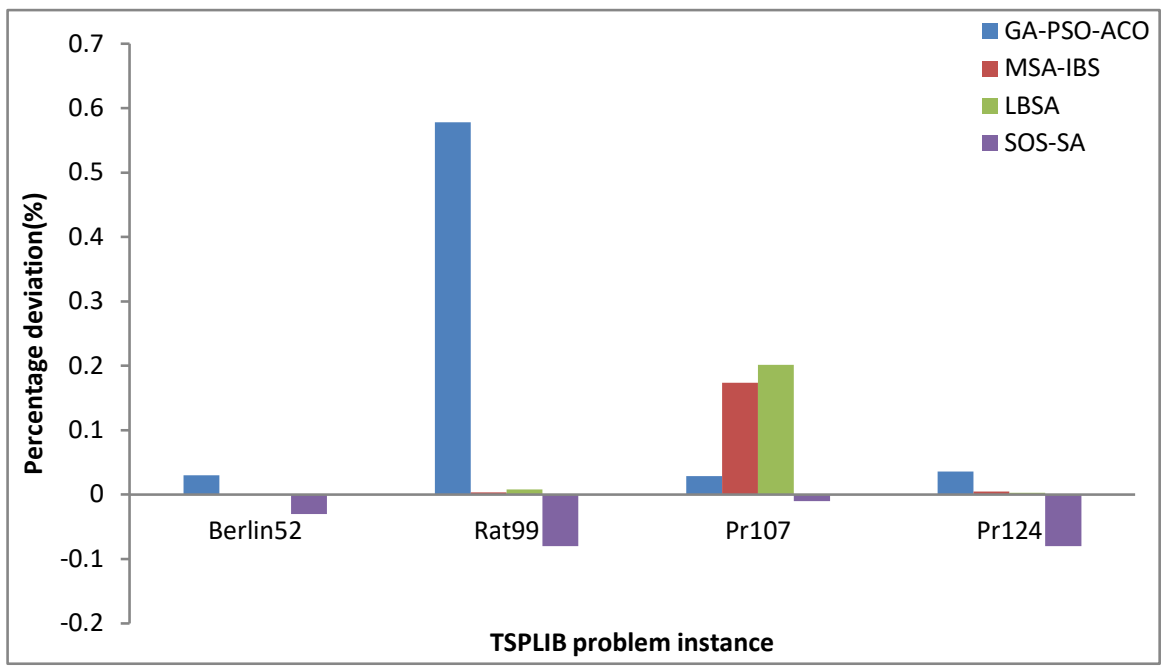

Fig.2. SOS-SA best solution percentage deviations compared with GA-PSO-ACO, MSA-IBS, and LBSA.

Tables 3, 4, 5, 6, 7, 8, and 9, demonstrate the comparisons among GA-PSO-ACO, MSA-IBS, LBSA, SOS, and SOS-SA algorithms on seven (7) different TSP instances. The comparisons are based on the quality of results produced by each of the algorithms. Also, as earlier stated, negative value means that the SOS-SA solution is better than the BKS solution.

Table 3: Algorithms comparison for Berlin52

\begin{tabular}{llllllll}
\hline Algorithm & Scale & BKS & Best & Mean & Diff. & PDbest (\%) & PDmean(\%) \\
\hline GA-PSO-ACO [Deng et al., 2012] & 52 & 7542 & 7544.37 & 7544.37 & 2.37 & 0.0314 & 0.0314 \\
MSA-IBS [Wang et al., 2015] & 52 & 7542 & 7542 & 7542 & 0 & 0 & 0 \\
LBSA [Zhan et al., 2016] & 52 & 7542 & 7542 & 7542 & 0 & 0 & 0 \\
SOS & 52 & 7542 & 7647 & 7659.48 & 104.68 & 1.3880 & 1.5577 \\
SOS-SA & 52 & 7542 & $\mathbf{7 5 4 0}$ & 7541.12 & -2 & -0.0265 & -0.0117 \\
\hline
\end{tabular}

Table 4: Algorithms comparison for Rat99

\begin{tabular}{llllllll}
\hline Algorithm & Scale & BKS & Best & Mean & Diff. & PDbest (\%) & PDmean(\%) \\
\hline GA-PSO-ACO [Deng et al., 2012] & 99 & 1211 & 1218 & 1275 & 7 & 0.5780 & 5.2849 \\
MSA-IBS [Wang et al., 2015] & 99 & 1211 & 1211 & 1211.04 & 0 & 0 & 0.0033 \\
LBSA [Zhan et al., 2016] & 99 & 1211 & 1211 & 1211.1 & 0 & 0 & 0.0083 \\
SOS & 99 & 1211 & 1284 & 1297.381 & 73 & 6.0281 & 7.1330 \\
SOS-SA & 99 & 1211 & $\mathbf{1 2 1 0}$ & 1210.108 & -1 & -0.0826 & -0.0737 \\
\hline \multicolumn{7}{c}{} \\
& Table 5: Algorithms comparison for Pr107 & & \\
\hline Algorithm & Scale & BKS & Best & Mean & Diff. & PDbest (\%) & PDmean(\%) \\
\hline GA-PSO-ACO [Deng et al., 2012] & 107 & 44303 & 44316 & 44589 & 13 & 0.0293 & 0.6456 \\
MSA-IBS [Wang et al., 2015] & 107 & 44303 & 44303 & 44379.88 & 0 & 0 & 0.1735 \\
LBSA [Zhan et al., 2016] & 107 & 44303 & 44303 & 44392.25 & 0 & 0 & 0.2015 \\
SOS & 107 & 44303 & 46097 & 46112.22 & 1794 & 4.0494 & 4.0837 \\
SOS-SA & 107 & 44303 & $\mathbf{4 4 3 0 1}$ & 44302.83 & -2 & -0.0045 & -0.0004 \\
\hline
\end{tabular}

Table 6: Algorithms comparison for Pr124

\begin{tabular}{llllllll}
\hline Algorithm & Scale & BKS & Best & Mean & Diff. & PDbest (\%) & PDmean(\%) \\
\hline GA-PSO-ACO [Deng et al., 2012] & 124 & 59030 & 59051 & 60157 & 21 & 0.0004 & 1.9092 \\
MSA-IBS [Wang et al., 2015] & 124 & 59030 & 59030 & 59032.88 & 0 & 0 & 0.0049 \\
LBSA [Zhan et al., 2016] & 124 & 59030 & 59030 & 59031.80 & 0 & 0 & 0.0030 \\
SOS & 124 & 59030 & 68942 & 69211.12 & 9912 & 0.1679 & 17.2474 \\
SOS-SA & 124 & 59030 & $\mathbf{5 8 9 8 5}$ & 59010.65 & -45 & -0.0008 & -0.0328 \\
\hline
\end{tabular}


Table 7: Algorithms comparison for Rat575

\begin{tabular}{llllllll}
\hline Algorithm & Scale & BKS & Best & Mean & Diff. & PDbest (\%) & PDmean(\%) \\
\hline GA-PSO-ACO [Deng et al., 2012] & 575 & 6773 & 6912 & 6952 & 139 & 2.0523 & 2.6428 \\
MSA-IBS [Wang et al., 2015] & 575 & 6773 & 6819 & 6854.64 & 46 & 0.6792 & 1.2054 \\
LBSA [Zhan et al., 2016] & 575 & 6773 & 6829 & 6847.95 & 56 & 0.8268 & 1.1066 \\
SOS & 575 & 6773 & 7018.22 & 6991.92 & 245.22 & 3.6206 & 3.2322 \\
SOS-SA & 575 & 6773 & 6773 & 6802.04 & 0 & 0 & 0.4288 \\
\hline
\end{tabular}

\begin{tabular}{llllllll}
\hline \multicolumn{7}{l}{ Table 8: Algorithms comparison for Rat783 } \\
\hline Algorithm & Scale & BKS & Best & Mean & Diff. & PDbest (\%) & PDmean(\%) \\
\hline GA-PSO-ACO [Deng et al., 2012] & 783 & 8806 & 9030 & 9126 & 224 & 2.5437 & 3.6339 \\
MSA-IBS [Wang et al., 2015] & 783 & 8806 & 8897 & 8918.28 & 91 & 1.0334 & 1.2750 \\
LBSA [Zhan et al., 2016] & 783 & 8806 & 8887 & 8913.25 & 81 & 0.9198 & 1.2179 \\
SOS & 783 & 8806 & 9884.37 & 9906.88 & 1078.37 & 12.2459 & 12.501 \\
SOS-SA & 783 & 8806 & 8806 & 8817.91 & 0 & 0 & 0.1353 \\
\hline
\end{tabular}

Table 9: Algorithms comparison for Pr1002

\begin{tabular}{llllllll}
\hline Algorithm & Scale & BKS & Best & Mean & Diff. & PDbest (\%) & PDmean(\%) \\
\hline GA-PSO-ACO [Deng et al., 2012] & 1002 & 259045 & 265987 & 266774 & 6942 & 2.6798 & 2.9837 \\
MSA-IBS [Wang et al., 2015] & 1002 & 259045 & 261463 & 262211.7 & 2418 & 0.9334 & 1.2225 \\
LBSA [Zhan et al., 2016] & 1002 & 259045 & 261490 & 262202.5 & 2445 & 0.9439 & 1.2189 \\
SOS & 1002 & 259045 & 280169.68 & 281140.08 & 21124.68 & 8.1548 & 8.5294 \\
SOS-SA & 1002 & 259045 & 261491 & 262301.8 & 2446 & 0.9442 & 1.2572 \\
\hline
\end{tabular}

In Table 10, the computational cost for the four algorithms are given in the last column under the title 'Time', and it's clear that the SOS-SA has the least convergence time frame compared to the other three algorithms. Generally, in terms of the convergence time, it can be argued that the SOS-SA is more successful than the remaining three algorithms, considering for example: the TSP instance 'Rd400' where it takes SOS-SA 2.71 seconds to converge, while in the case of the other three, it took 30.4 seconds for ASA-GS, 3.2 seconds for MSA-IBS, and 3.46 seconds for LBSA respectively. However, there are instances where the SOS-SA is outperformed by both LBSA and MSA-IBS, and this could be attributed to the deep explorative and exploitative capability of the SOS component in SOS-SA algorithm that would at times incur additional cost in finding global best tour routes. One example of this case can be seen in the problem instance 'D1291' where MSA-IBS with convergence time of 10.59 outperformed both LBSA and SOS-SA algorithms, with each having convergence time of 11.77 and 12.08 respectively. This exceptional behaviour exhibited at times by MSA-IBS can be traced to some level of intelligence acquired from the learning-based sampling process, which can effectively improve the performance of the SA's sampling efficiency. Figs. 3-5 show the different convergence rates for the four algorithms with regards to varying TSP problem lengths.

Table 10: ASA-GS, MSA-IBS, LBSA, and SOS-SA convergence times and speed comparison, the results are the average of 1000 executions. The best results are highlighted in bold.

\begin{tabular}{|c|c|c|c|c|c|c|c|c|c|c|}
\hline \multirow{2}{*}{$S / N$} & \multirow{2}{*}{ Instance } & \multirow{2}{*}{ BKS } & \multicolumn{2}{|c|}{ ASA-GS [Geng et al., 2011] } & \multicolumn{2}{|c|}{ MSA-IBS [Wang et al., 2015] } & \multicolumn{2}{|c|}{ LBSA [Zhan et al., 2016] } & \multicolumn{2}{|c|}{ SOS-SA } \\
\hline & & & Mean & Time & Mean & Time & Mean & Time & Mean & Time \\
\hline 1 & Ch150 & 6528 & 6539.8 & 10.91 & 6529 & 0.86 & 6529.8 & 1.29 & 6529.8384 & 1.0296 \\
\hline 2 & Kroa150 & 26524 & 26538.6 & 10.9 & 26524 & 0.82 & 26524 & 0.98 & 26524.0176 & 0.7020 \\
\hline 3 & Krob150 & 26130 & 26178.1 & 10.9 & 26135 & 1.51 & 26137 & 1.65 & 26131.8331 & 1.1700 \\
\hline 4 & Pr152 & 73682 & 73694.7 & 10.85 & 73682 & 0.84 & 73682 & 0.87 & 73682.1801 & 0.8268 \\
\hline 5 & U159 & 42080 & 42398.9 & 11.49 & 42080 & 0.79 & 42080 & 0.91 & 42080.9819 & 0.8736 \\
\hline 6 & Rat195 & 2323 & 2348.05 & 14.37 & 2330.2 & 1.86 & 2328 & 1.93 & 2326.5979 & 1.1856 \\
\hline 7 & D198 & 15780 & 15845.4 & 14.6 & 15780 & 1.39 & 15780 & 1.53 & 15782.1061 & 1.0452 \\
\hline 8 & Kroa200 & 29368 & 29438.4 & 14.26 & 29378 & 1.74 & 29373.8 & 1.67 & 29370.7811 & 1.2792 \\
\hline 9 & Krob200 & 29437 & 29513.1 & 14.24 & 29439.8 & 1.95 & 29442.2 & 2.1 & 29449.8182 & 1.5132 \\
\hline 10 & $\begin{array}{l}\text { Ts225 } \\
\end{array}$ & 126643 & 126646 & 16.05 & 126643 & 1.3 & 126643 & 1.54 & 126701.0841 & 1.4196 \\
\hline 11 & Pr226 & 80369 & 80687.4 & 16.7 & 80369 & 1.93 & 80369.8 & 2.16 & 80369.3077 & 1.5444 \\
\hline 12 & Gil262 & 2378 & 2398.61 & 19.43 & 2378.8 & 2.39 & 2379.2 & 2.72 & 2381.9145 & 2.0387 \\
\hline 13 & Pr264 & 49135 & 49138.9 & 19.09 & 49135 & 1.43 & 49135 & 1.49 & 49135.7188 & 1.5056 \\
\hline 14 & Pr299 & 48191 & 48326.4 & 21.94 & 48226.4 & 2.67 & 48221.2 & 2.93 & 48227.9301 & 2.3444 \\
\hline 15 & Lin318 & 42029 & 42383.7 & 23.35 & 42184.4 & 2.4 & 42195.6 & 2.58 & 42179.3111 & 2.6121 \\
\hline 16 & $\mathrm{Rd} 400$ & 15281 & 15429.8 & 30.4 & 15347.2 & 3.2 & 15350.4 & 3.46 & 15451.8108 & 2.7114 \\
\hline 17 & Fl417 & 11861 & 12043.8 & 32.02 & 11875.6 & 3.72 & 11867.8 & 4.01 & 11877.5194 & 3.9410 \\
\hline 18 & Pr439 & 107217 & 110226 & 34.92 & 107407.2 & 3.6 & 107465.2 & 3.95 & 107561.1441 & 3.1801 \\
\hline 19 & Pcb442 & 50778 & 51269.2 & 35.75 & 50970 & 3.68 & 50877 & 4.31 & 50871.8228 & 4.4017 \\
\hline 20 & U574 & 36905 & 37369.8 & 48.47 & 37155.8 & 5.21 & 37164.6 & 6.13 & 37164.4871 & 6.6099 \\
\hline 21 & Rat575 & 6773 & 6904.82 & 52.1 & 6839.8 & 5.27 & 6837.4 & 5.99 & 6839.5194 & 5.1184 \\
\hline 22 & U724 & 41910 & 42470.4 & 66.83 & 42212.2 & 8.11 & 42252 & 8.34 & 42262.1108 & 7.9999 \\
\hline 23 & Rat783 & 8806 & 8982.19 & 78.9 & 8893.4 & 8.99 & 8888.2 & 8.9 & 8899.5507 & 8.6130 \\
\hline 24 & Pr1002 & 259045 & 264274 & 164.42 & 261481.8 & 12.71 & 261805.2 & 12.96 & 261802.4892 & 12.8141 \\
\hline 25 & Pcb1173 & 56892 & 57820.5 & 193.08 & 57561.6 & 8.9 & 57431.8 & 9.61 & 57569.9388 & 8.7301 \\
\hline 26 & D1291 & 50801 & 52252.3 & 214.64 & 51343.8 & 10.59 & 51198.8 & 11.77 & 51291.0871 & 12.0816 \\
\hline 27 & Rl1323 & 270199 & 273444 & 210.16 & 271818.4 & 11.53 & 271714.4 & 12.64 & 271710.6288 & 11.0188 \\
\hline 28 & Fl1400 & 20127 & 20782.2 & 232.02 & 20374.8 & 17.72 & 20249.4 & 15.43 & 20231.0177 & 14.7381 \\
\hline 29 & D1655 & 62128 & 64155.9 & 281.88 & 62893 & 16.18 & 63001.4 & 16.45 & 64111.9201 & 16.1902 \\
\hline 30 & Vm1748 & 336556 & 343911 & 276.98 & 339617.8 & 19.7 & 339710.8 & 19.05 & 336719.3891 & 18.2714 \\
\hline 31 & U2319 & 234256 & 236744 & 410.97 & 235236 & 17.02 & 235975 & 18.94 & 235338.0944 & 18.1111 \\
\hline 32 & Pcb3038 & 137694 & 141242 & 554.28 & 139706.2 & 27.64 & 139635.2 & 29.05 & 139701.8133 & 25.6712 \\
\hline
\end{tabular}




\begin{tabular}{|c|c|c|c|c|c|c|c|c|c|c|}
\hline 33 & Fnl4461 & 182566 & 187409 & 830.9 & 185535.4 & 30.43 & 185509.4 & 29.67 & 185546.0411 & 32.7422 \\
\hline 34 & RI5934 & 556045 & 575437 & 1043.95 & 566166.8 & 50.76 & 566053 & 52.5 & 566211.7184 & 49.9871 \\
\hline 35 & Pla7397 & 23260728 & 24166453 & 1245.22 & $2.38 \mathrm{E}+07$ & 100.69 & $2.38 \mathrm{E}+07$ & 89.61 & $2.38 \mathrm{E}+07$ & 98.7222 \\
\hline 36 & Usa13509 & 19982859 & 20811106 & 2016.05 & $2.04 \mathrm{E}+07$ & 365.12 & $2.04 \mathrm{E}+07$ & 326.76 & $2.14 \mathrm{E}+07$ & 313.1080 \\
\hline 37 & Brd14051 & 469385 & 486197 & 2080.5 & 478609.6 & 375.28 & 478010 & 369.86 & 478098.9076 & 370.8801 \\
\hline 38 & D18512 & 645238 & 669445 & 2593.97 & 658149.2 & 654.85 & 657457.2 & 629.14 & 659457.4512 & 601.8544 \\
\hline 39 & Pla33810 & 66048945 & 69533166 & 4199.88 & 68075607 & 1959.68 & 68029226.4 & 1998.19 & 68076220.2281 & 1899.9919 \\
\hline \multirow{2}{*}{40} & Pla85900 & 142382641 & 156083025 & 8855.13 & 146495515.6 & 7596.18 & 145526542.6 & 7586.6 & 146429581.1412 & 7591.1833 \\
\hline & & Average & & 650.31 & & 283.52 & & 282.49 & & 278.99403 \\
\hline
\end{tabular}

Overall, the SOS-SA performed better than the remaining three algorithms in $62.5 \%$ of the computed graphs ranging from 150 up to 85,900 cities (i.e., 25 out of 40 instances).

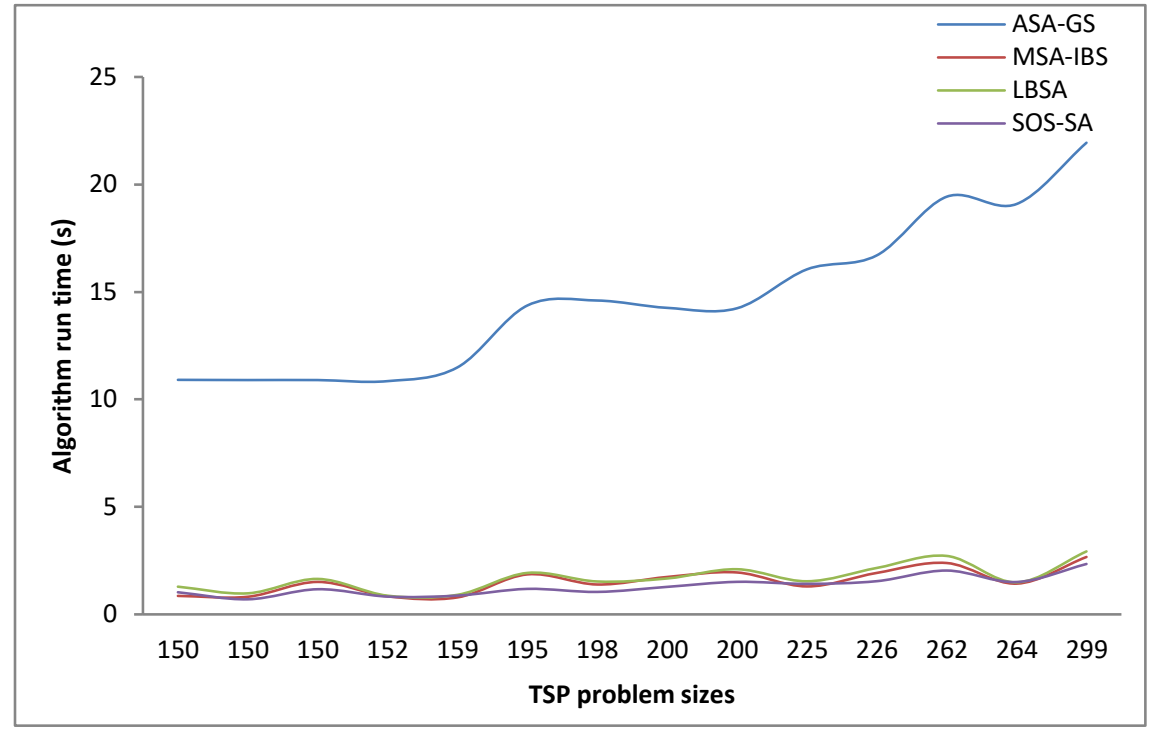

Fig.3. ASA-GS, MSA-IBS, LBSA, and SOS-SA time comparison, for TSP with graphs ranging from 150 up to 299 cities

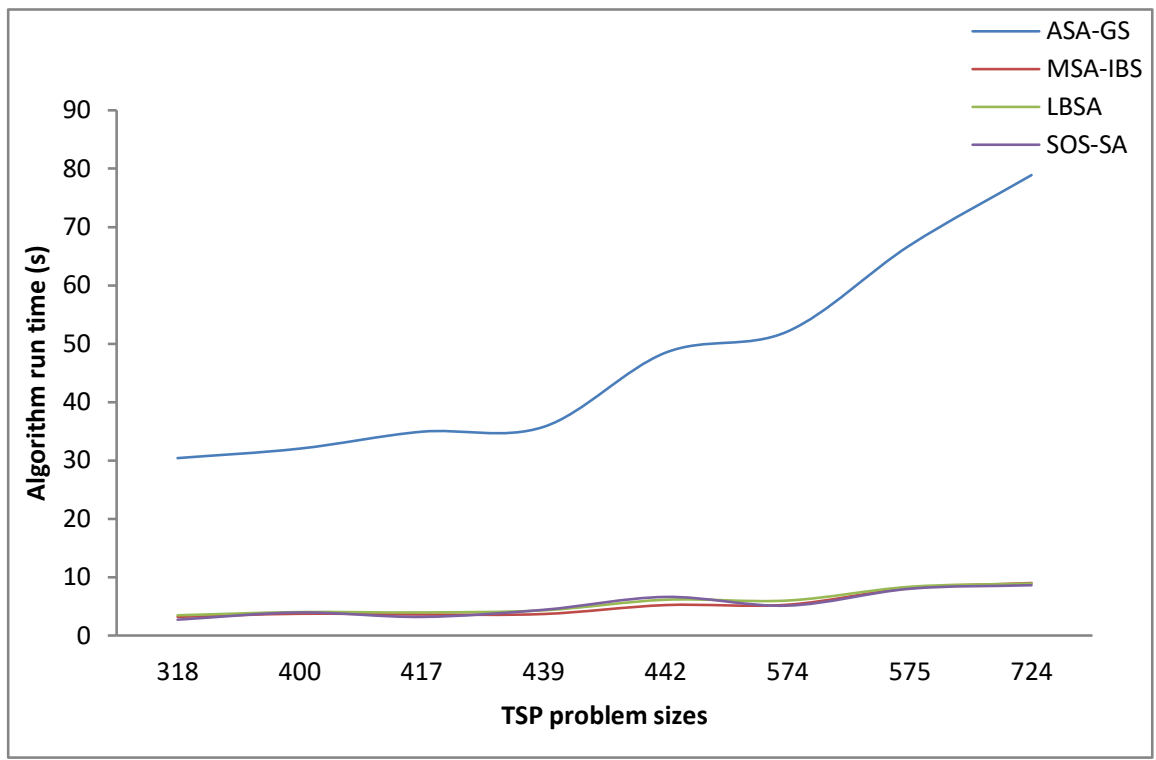

Fig.4. ASA-GS, MSA-IBS, LBSA, and SOS-SA time comparison, for TSP with graphs ranging from 318 up to 724 cities. 


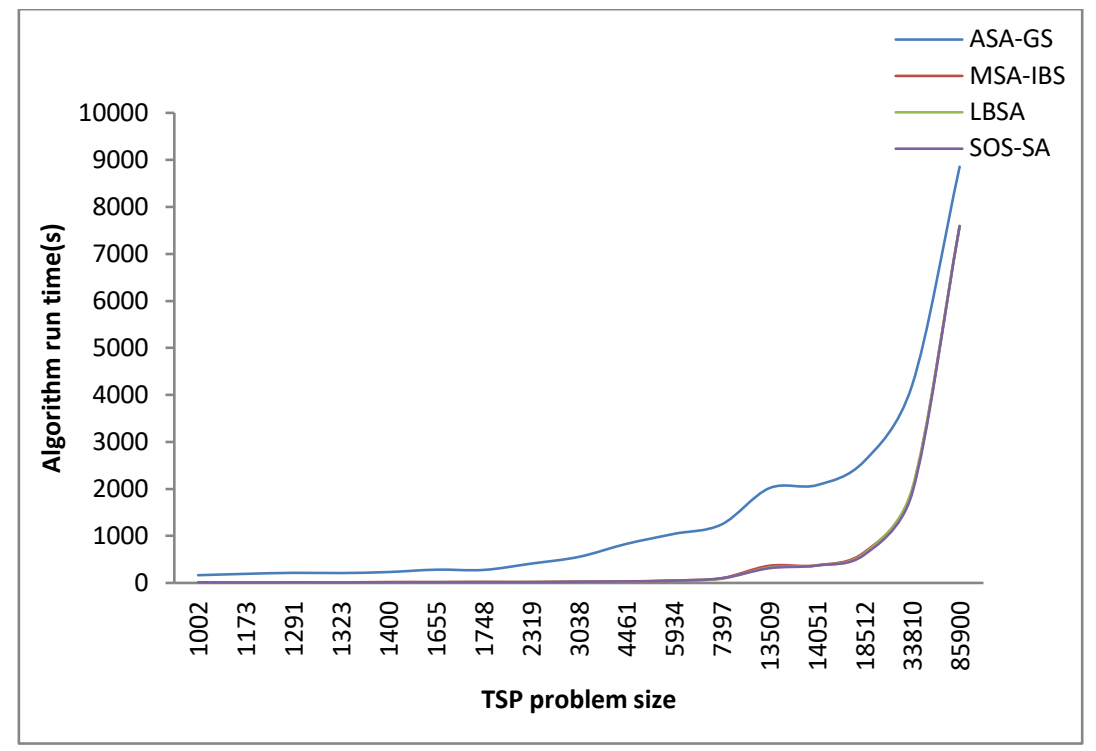

Fig.5. ASA-GS, MSA-IBS, LBSA, and SOS-SA time comparison, for TSP with graphs ranging from 1002 up to 85,900 cities.

In Table 11, the percentage deviation of the SOS-SA for the 35 TSP instance considered was computed to be 0.2645 , which is significantly better than the 1.2929 of GA-PSO-ACO, 0.3385 of MSA-IBS, and 0.3229 of LBSA respectively. Therefore, this verifies the fact that the performance of the SOS-SA algorithms competes favourably with the state-of-the-art TSP algorithms and in some instance with the best known solution from the TSPLIB problem instances. 
Table 11: GA-PSO-ACO, MSA-IBS, LBSA, and SOS-SA comparison on 35 benchmarked TSPLIB tour instances for 20 trials. The columns shows the best solution found, the average solution and the relative percentage error computed using Eq. (10). The best results so far are highlighted in bold.

\begin{tabular}{|c|c|c|c|c|c|c|c|c|c|c|c|c|c|c|}
\hline $\mathbf{S} / \mathbf{N}$ & Instance & BKS & GA-PSO-A & $\mathbf{O}$ [Deng $e$ & l., 2012] & MSA- & $3 \mathbf{S}$ [Wang $e t$ & $l l ., 2015]$ & $\mathbf{L B}$ & [Zhan et al & 2016] & & SOS-SA & \\
\hline & & & Best & Mean & $\begin{array}{c}\text { PDbest } \\
(\%)\end{array}$ & Best & Mean & PDbest (\%) & Best & Mean & PDbest (\%) & Best & Mean & PDbest (\%) \\
\hline 1 & Att48 & 33522 & 33524 & 33662 & 0.0060 & 33522 & 33554.64 & 0 & 33522 & 33536.6 & 0 & 33523 & 33539.68 & 0.0030 \\
\hline 2 & Eli51 & 426 & 426 & 431.84 & 0 & 426 & 426.48 & 0 & 426 & 426.5 & 0 & 426 & 426 & 0 \\
\hline 3 & Berlin52 & 7542 & 7544.37 & 7544.37 & 0.0314 & 7542 & 7542 & 0 & 7542 & 7542 & 0 & 7540 & 7541.107 & -0.0265 \\
\hline 4 & St70 & 675 & 679.6 & 694.6 & 0.6815 & 675 & 677.16 & 0 & 675 & 675.55 & 0 & 675 & 675 & 0 \\
\hline 5 & Eil76 & 538 & 545.39 & 550.16 & 1.3736 & 538 & 538.2 & 0 & 538 & 538 & 0 & 538 & 538 & 0 \\
\hline 6 & Pr76 & $\begin{array}{c}10815 \\
9 \\
\end{array}$ & 109206 & 110023 & 0.9680 & 108159 & 108288 & 0 & 108159 & 108268.3 & 0 & 108162 & 109214.8 & 0.0028 \\
\hline 7 & Rat99 & 1211 & 1218 & 1275 & 0.5780 & 1211 & 1211.04 & 0 & 1211 & 1211.1 & 0 & 1210 & 1210.108 & -0.0826 \\
\hline 8 & Rad100 & 7910 & 7936 & 8039 & 0.3287 & 7910 & 7914.56 & 0 & 7910 & 7914.7 & 0 & 7910 & 7912.875 & 0 \\
\hline 9 & KroD100 & 21294 & 21309 & 21484 & 0.0704 & 21294 & 21340.64 & 0 & 21294 & 21314.2 & 0 & 21294 & 21410.02 & 0 \\
\hline 10 & Eil101 & 629 & 633.07 & 637.93 & 0.6471 & 629 & 629.6 & 0 & 629 & 629 & 0 & 629 & 629 & 0 \\
\hline 11 & Lin105 & 14379 & 14397 & 14521 & 0.1252 & 14379 & 14380.48 & 0 & 14379 & 14379 & 0 & 14379 & 14380.27 & 0 \\
\hline 12 & Pr107 & 44303 & 44316 & 44589 & 0.0293 & 44303 & 44379.88 & 0 & 44303 & 44392.25 & 0 & 44301 & 44302.83 & -0.0045 \\
\hline 13 & Pr124 & 59030 & 59051 & 60157 & 0.0356 & 59030 & 59032.88 & 0 & 59030 & 59031.8 & 0 & 58985 & 59010.65 & -0.0762 \\
\hline 14 & Bier127 & $\begin{array}{c}11828 \\
2 \\
\end{array}$ & 118282 & 120301 & 0 & 118282 & 118334.6 & 0 & 118282 & 118351.2 & 0 & 118282 & 118331.2 & 0 \\
\hline 15 & Ch130 & 6110 & 6141 & 6203.47 & 0.5074 & 6110 & 6121.96 & 0 & 6110 & 6127.95 & 0 & 6110 & 6110 & 0 \\
\hline 16 & Pr144 & 58537 & 58595 & 58662 & 0.0991 & 58537 & 58549.72 & 0 & 58537 & 58570.15 & 0 & 58537 & 58610.91 & 0 \\
\hline 17 & KroA150 & 26524 & 26676 & 26803 & 0.5731 & 26524 & 26538.2 & 0 & 26524 & 26542.6 & 0 & 26524 & 27032.18 & 0 \\
\hline 18 & Pr152 & 73682 & 73861 & 73989 & 0.2429 & 73682 & 73727.96 & 0 & 73682 & 73688.8 & 0 & 73683 & 73689.56 & 0.0014 \\
\hline 19 & U159 & 42080 & 42395 & 42506 & 0.7486 & 42080 & 42182.32 & 0 & 42080 & 42198.85 & 0 & 42080 & 42188.47 & 0 \\
\hline 20 & Rat195 & 2323 & 2341 & 2362 & 0.7749 & 2328 & 2334.2 & 0.2152 & 2328 & 2331 & 0.2152 & 2325 & 2329.032 & 0.0861 \\
\hline 21 & RroA200 & 29368 & 29731 & 31015 & 1.2360 & 29368 & 29422.64 & 0 & 29368 & 29405.35 & 0 & 29368 & 29435.76 & 0 \\
\hline 22 & Gil262 & 2378 & 2399 & 2439 & 0.8831 & 2379 & 2383.56 & 0.0421 & 2379 & 2382.45 & 0.0421 & 2381 & 2384.695 & 0.1262 \\
\hline 23 & $\operatorname{Pr} 299$ & 48191 & 48662 & 48763 & 0.9774 & 48192 & 48263.08 & 0.0021 & 48191 & 48250 & 0 & 48191 & 48197.49 & 0 \\
\hline
\end{tabular}




\begin{tabular}{|c|c|c|c|c|c|c|c|c|c|c|c|c|c|c|}
\hline 24 & $\operatorname{Lin} 318$ & 42029 & 42633 & 42771 & 1.4371 & 42076 & 42292.04 & 0.1118 & 42070 & 42264.35 & 0.0976 & 42029 & 42291.67 & 0 \\
\hline 25 & $\mathrm{Rd} 400$ & 15281 & 15464 & 15503 & 1.19757 & 15324 & 15377.56 & 0.2814 & 15311 & 15373.75 & 0.1963 & 15310 & 15318.11 & 0.1898 \\
\hline 26 & Pcb442 & 50778 & 51414 & 51494 & 1.2525 & 50879 & 51050.12 & 0.1989 & 50832 & 51041.7 & 0.1063 & 50812 & 51039.21 & 0.0670 \\
\hline 27 & Rat575 & 6773 & 6912 & 6952 & 2.0523 & 6819 & 6854.64 & 0.6792 & 6829 & 6847.95 & 0.8268 & 6773 & 6802.04 & 0 \\
\hline 28 & U724 & 41910 & 42657 & 42713 & 1.7824 & 42150 & 42302.12 & 0.5727 & 42205 & 42357.8 & 0.7039 & 41910 & 42262.11 & 0 \\
\hline 29 & Rat783 & 8806 & 9030 & 9126 & 2.5437 & 8897 & 8918.28 & 1.0334 & 8887 & 8913.25 & 0.9198 & 8806 & 8817.91 & 0 \\
\hline 30 & Pr1002 & $\begin{array}{c}25904 \\
5\end{array}$ & 265987 & 266774 & 2.6798 & 261463 & 262211.7 & 0.9334 & 261490 & 262202.5 & 0.9439 & 261491 & 262301.8 & 0.9442 \\
\hline 31 & D1291 & 50801 & 52378 & 52443 & 3.1043 & 51098 & 51340.84 & 0.5846 & 51032 & 51358.7 & 0.4547 & 51091 & 51316.18 & 0.5709 \\
\hline 32 & D1655 & 62128 & 64401 & 65241 & 3.6586 & 62784 & 63011.96 & 1.0559 & 62779 & 62994.65 & 1.0478 & 62779 & 63014.18 & 1.0478 \\
\hline 33 & $\mathrm{~N} \mid 4461$ & $\begin{array}{c}18256 \\
6 \\
\end{array}$ & 189334 & 192574 & 3.7072 & 185377 & 185631.1 & 1.5397 & 185290 & 185501.7 & 1.4921 & 185361 & 185401.7 & 1.5310 \\
\hline 34 & Brd14051 & $\begin{array}{c}66938 \\
5 \\
\end{array}$ & 490432 & 503560 & 4.4840 & 478040 & 478618.8 & 1.8439 & 477226 & 477612.7 & 1.6705 & 478385 & 477817 & 1.9174 \\
\hline 35 & Pla33810 & $\begin{array}{c}66048 \\
945\end{array}$ & 70299195 & 72420147 & 6.4350 & 67868250 & 68038833 & 2.7545 & 67754877 & 67848535 & 2.5828 & 68004101 & 67100510 & 2.9602 \\
\hline \multicolumn{5}{|c|}{ Average values: } & 1.2929 & & & 0.3385 & & & 0.3229 & & & 0.2645 \\
\hline
\end{tabular}


In Tables 12 and 13, the comparisons of SOS-SA, SOS, and IBA are presented. The computed average values for the PDbest and PDmean of SOS-SA were obtained as -0.1262 and 0.4230 as shown in Table 12, and 0.0262 and 0.4891 as shown in Table 13. The average values for the SOS were obtained as 2.5019 and 4.3537 respectively, while those of the IBA in Table 13 were obtained as 0.2106 and 1.3911 respectively. Therefore, comparing the three algorithms, the computed average values for PDbest and PDmean show that SOS-SA obtained smaller percentage deviations than the SOS and IBA algorithms in all the instances considered in the two tables.

The percentage deviation test carried out shows the capability of the SOS-SA algorithm to find the best solution in a more effective and efficient manner. This can be attributed to the hybridization characteristics of the individual algorithms, where the systematic reasoning skill of the SA based on its ability to use the acceptance probability criteria to find better solution within the problem local search space is added to the exploration and exploitation capability of the SOS algorithm.

Table12: Comparison of SOS-SA with SOS, where PDbest and PDmean represent the percentage deviations of both the best solution found and the average solution to the best known solution (BKS).

\begin{tabular}{|c|c|c|c|c|c|c|c|c|c|c|c|c|}
\hline \multirow[t]{2}{*}{$S / N$} & \multirow{2}{*}{$\begin{array}{l}\text { Instance } \\
\text { Name }\end{array}$} & \multirow[b]{2}{*}{ BKS } & \multicolumn{3}{|c|}{ SOS-SA } & \multirow[b]{2}{*}{ PDbest (\%) } & \multirow[b]{2}{*}{ PDmean(\%) } & \multicolumn{5}{|c|}{ SOS } \\
\hline & & & Mean & Best & Diff. & & & Mean & Best & Diff. & PDbest (\%) & PDmean(\%) \\
\hline 1 & dantzig42 & 699 & 699.482 & 679 & -20 & -2.8612 & 0.0690 & 710.502 & 702 & 3 & 0.4292 & 1.6455 \\
\hline 2 & Eil51 & 426 & 426 & 426 & 0 & 0 & 0 & 438.728 & 429 & 3 & 0.7042 & 2.9878 \\
\hline 3 & Berlin52 & 7542 & 7541.11 & 7540 & -2 & -0.0265 & -0.0118 & 7659.49 & 7647 & 105 & 1.3922 & 1.5578 \\
\hline 4 & St70 & 675 & 675 & 675 & 0 & 0 & 0 & 699.129 & 675 & 0 & 0 & 3.5747 \\
\hline 5 & Eil76 & 538 & 538 & 538 & 0 & 0 & 0 & 556.312 & 542 & 4 & 0.7435 & 3.4037 \\
\hline 6 & Rat99 & 1211 & 1210.11 & 1210 & -1 & -0.0826 & -0.0735 & 1297.38 & 1284 & 73 & 6.0281 & 7.1329 \\
\hline 7 & KroA100 & 21282 & 21424 & 21282 & 0 & 0 & 0.6672 & 21633.8 & 21401 & 119 & 0.5592 & 1.6530 \\
\hline 8 & KroB100 & 22140 & 22331.8 & 22140 & 0 & 0 & 0.8663 & 23142.8 & 22155 & 15 & 0.0678 & 4.5294 \\
\hline 9 & KroC100 & 20749 & 20860.8 & 20749 & 0 & 0 & 0.5388 & 21020.2 & 20811 & 62 & 0.2988 & 1.3071 \\
\hline 10 & KroD100 & 21294 & 21494.1 & 21294 & 0 & 0 & 0.9397 & 22044.3 & 21492 & 198 & 0.9298 & 3.5235 \\
\hline 11 & KroE100 & 22068 & 22205.9 & 22068 & 0 & 0 & 0.6249 & 22467.1 & 22128 & 60 & 0.2719 & 1.8085 \\
\hline 12 & Eil101 & 629 & 629 & 629 & 0 & 0 & 0 & 659.713 & 649 & 20 & 3.1797 & 4.8830 \\
\hline 13 & Pr107 & 44303 & 44302.8 & 44301 & -2 & -0.0045 & -0.0005 & 46112.2 & 46097 & 1794 & 4.0494 & 4.0837 \\
\hline 14 & Pr124 & 59030 & 59010.7 & 58985 & -45 & -0.0762 & -0.0327 & 69211.1 & 68942 & 9912 & 16.7915 & 17.2473 \\
\hline 15 & Pr136 & 96772 & 98636 & 97129 & 357 & 0.3689 & 1.9262 & 100461 & 98018 & 1246 & 1.2876 & 3.8121 \\
\hline 16 & Pr144 & 58537 & 58610.8 & 58537 & 0 & 0 & 0.1261 & 60136.9 & 58587 & 50 & 0.0854 & 2.7331 \\
\hline 17 & Pr152 & 73682 & 73689.6 & 73683 & 1 & 0.0014 & 0.0103 & 74699.8 & 74229 & 547 & 0.7424 & 1.3813 \\
\hline 18 & Pr264 & 49135 & 50201.6 & 49212 & 77 & 0.1567 & 2.1708 & 52498.5 & 51477 & 2342 & 4.7665 & 6.8454 \\
\hline 19 & Pr299 & 48191 & 48197.5 & 48191 & 0 & 0 & 0.0135 & 50102.4 & 49624 & 1433 & 2.9736 & 3.9663 \\
\hline 20 & Lin318 & 42029 & 42291.7 & 42029 & 0 & 0 & 0.6250 & 45811.1 & 44020 & 1991 & 4.7372 & 8.99879 \\
\hline & & & & verage V & & -0.1262 & 0.4230 & & & & 2.5019 & 4.3537 \\
\hline
\end{tabular}

Table 13: Table12: Comparison of SOS-SA with IBA, where PDbest and PDmean represent the percentage deviations of both the best solution found and the average solution to the best known solution (BKS).

\begin{tabular}{|c|c|c|c|c|c|c|c|c|c|c|c|c|}
\hline \multirow[t]{2}{*}{$S / N$} & \multirow{2}{*}{$\begin{array}{l}\text { Instance } \\
\text { Name }\end{array}$} & \multicolumn{6}{|c|}{ SOS-SA } & \multicolumn{5}{|c|}{ IBA [Osaba et al., 2016] } \\
\hline & & BKS & Mean & Best & Diff. & PDbest (\%) & PDmean(\%) & Mean & Best & Diff. & PDbest(\%) & PDmean(\%) \\
\hline 1 & Eil51 & 426 & 426 & 426 & 0 & 0 & 0 & 428.1 & 426 & 0 & 0 & 0.4929 \\
\hline 2 & Berlin52 & 7542 & 7541.11 & 7540 & -2 & -0.0265 & -0.0118 & 7542 & 7542 & 0 & 0 & 0 \\
\hline 3 & St70 & 675 & 675 & 675 & 0 & 0 & 0 & 679.1 & 675 & 0 & 0 & 0.6074 \\
\hline 4 & Eil76 & 538 & 538 & 538 & 0 & 0 & 0 & 548.1 & 539 & 1 & 0.1859 & 1.8773 \\
\hline 5 & KroA100 & 21282 & 21424 & 21282 & 0 & 0 & 0.6672 & 21445.3 & 21282 & 0 & 0 & 0.7673 \\
\hline
\end{tabular}




\begin{tabular}{|c|c|c|c|c|c|c|c|c|c|c|c|c|}
\hline 6 & KroB100 & 22140 & 22331.8 & 22140 & 0 & 0 & 0.8663 & 22506.4 & 22140 & 0 & 0 & 1.6549 \\
\hline 7 & KroC100 & 20749 & 20860.8 & 20749 & 0 & 0 & 0.5388 & 21050 & 20749 & 0 & 0 & 1.4507 \\
\hline 8 & KroD100 & 21294 & 21494.1 & 21294 & 0 & 0 & 0.9397 & 21593.4 & 21294 & 0 & 0 & 1.4060 \\
\hline 9 & KroE100 & 22068 & 22205.9 & 22068 & 0 & 0 & 0.6249 & 22349.6 & 22068 & 0 & 0 & 1.2761 \\
\hline 10 & Eil101 & 629 & 629 & 629 & 0 & 0 & 0 & 646.4 & 634 & 5 & 0.7949 & 2.7663 \\
\hline 11 & Pr107 & 44303 & 44302.8 & 44301 & -2 & -0.0045 & -0.0005 & 44793.8 & 44303 & 0 & 0 & 1.1078 \\
\hline 12 & Pr124 & 59030 & 59010.7 & 58985 & -45 & -0.0762 & -0.0327 & 59412.1 & 59030 & 0 & 0 & 0.6473 \\
\hline 13 & Pr136 & 96772 & 98636 & 97129 & 357 & 0.3689 & 1.9262 & 99351.2 & 97547 & 775 & 0.8009 & 2.6652 \\
\hline 14 & Pr144 & 58537 & 58610.8 & 58537 & 0 & 0 & 0.1261 & 58876.2 & 58537 & 0 & 0 & 0.5795 \\
\hline 15 & Pr152 & 73682 & 73689.6 & 73683 & 1 & 0.0014 & 0.0103 & 74676.9 & 73921 & 239 & 0.3244 & 1.3503 \\
\hline 16 & Pr264 & 49135 & 50201.6 & 49212 & 77 & 0.1567 & 2.1708 & 50908.3 & 49756 & 621 & 1.2639 & 3.6090 \\
\hline \multicolumn{6}{|c|}{ Average values: } & 0.0262 & 0.4891 & & & & 0.2106 & 1.3911 \\
\hline
\end{tabular}

Figs. 6-9, demonstrate the simulation tests of a number of TSB problem instances of different scales which were benchmarked to test the effectiveness of the new SOS-SA algorithm. The simulation results show route tracing and convergence graphs of each of the cities using SOS-SA algorithm. The algorithm execution was terminated after 2000 runs for each TSP instance. However, we are only able to show convergence graphs for the proposed algorithm because the other compared algorithms' data were taken from literature and this information was unavailable.
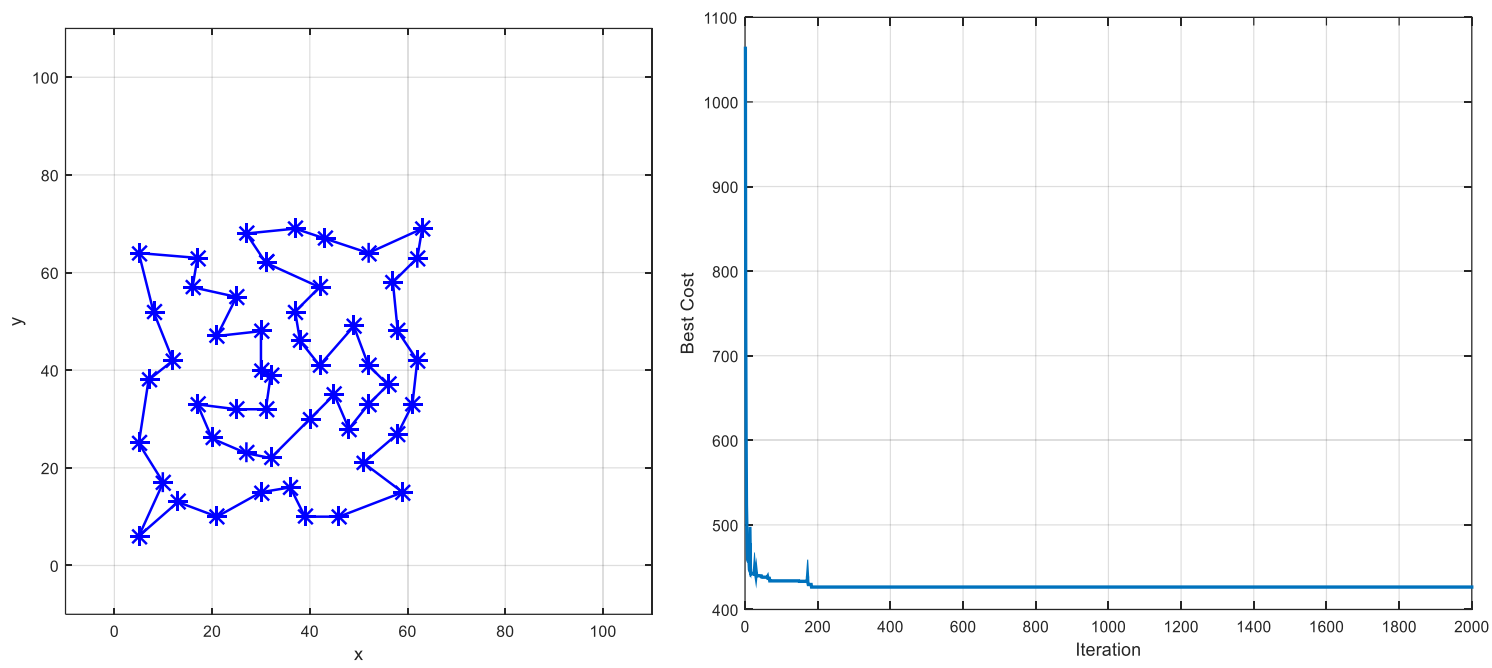

Fig.6. the result of 52-city (Eli52) TSPs using SOS-SA
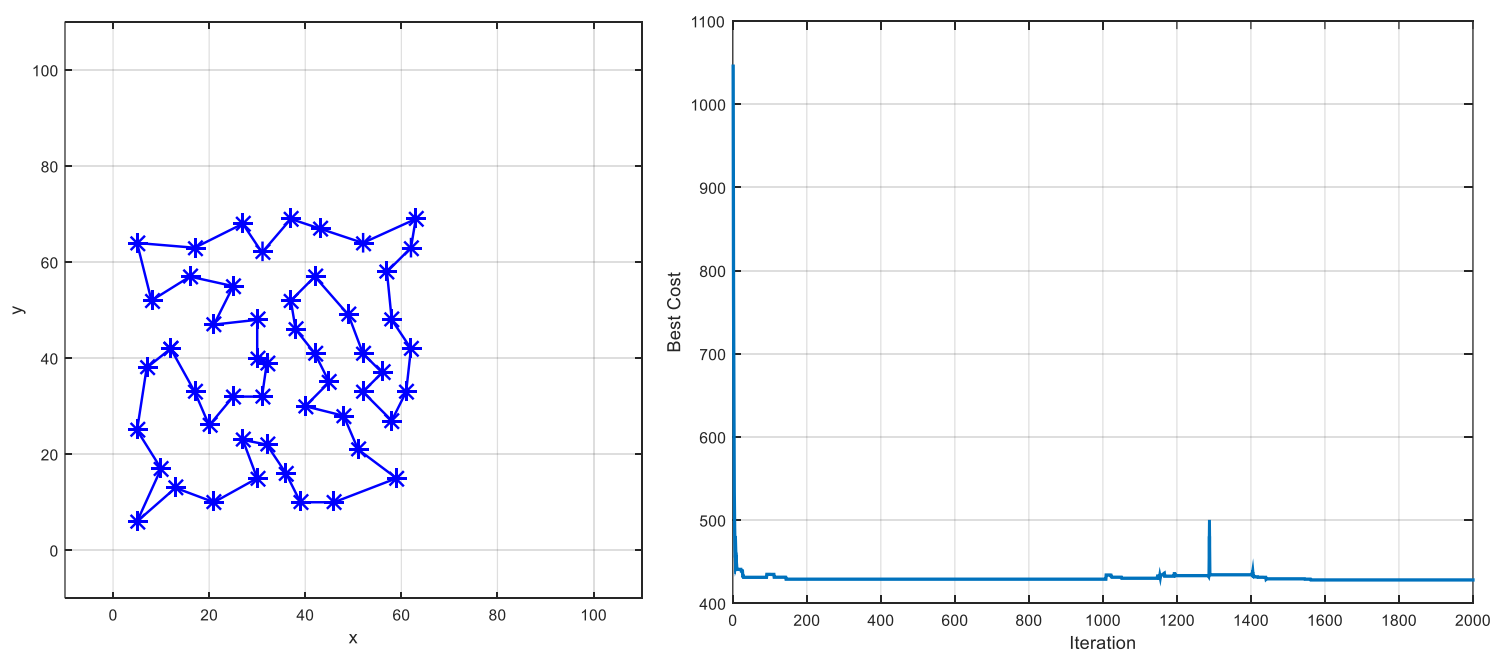

Fig.7. the result of 54-city (Eil54) TSPs using SOS-SA 

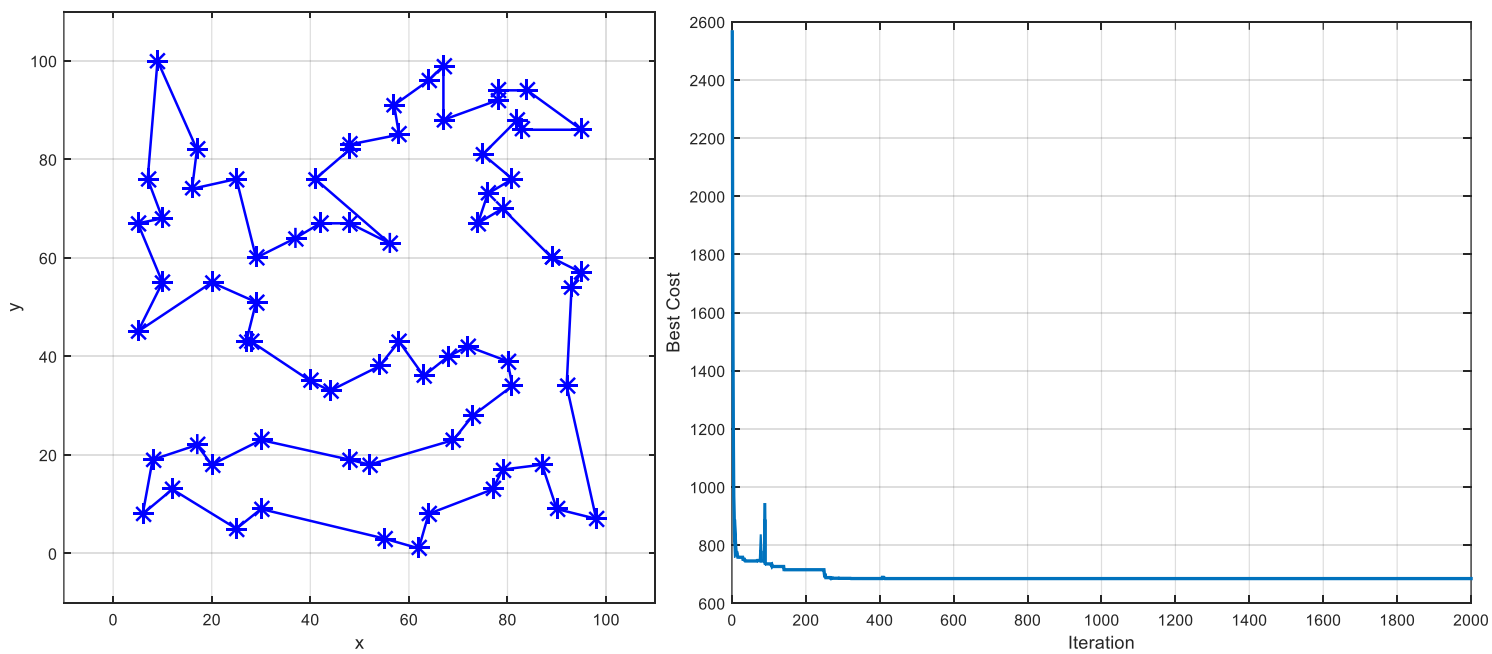

Fig.8. the result of 70-city (St70) TSPs using SOS-SA
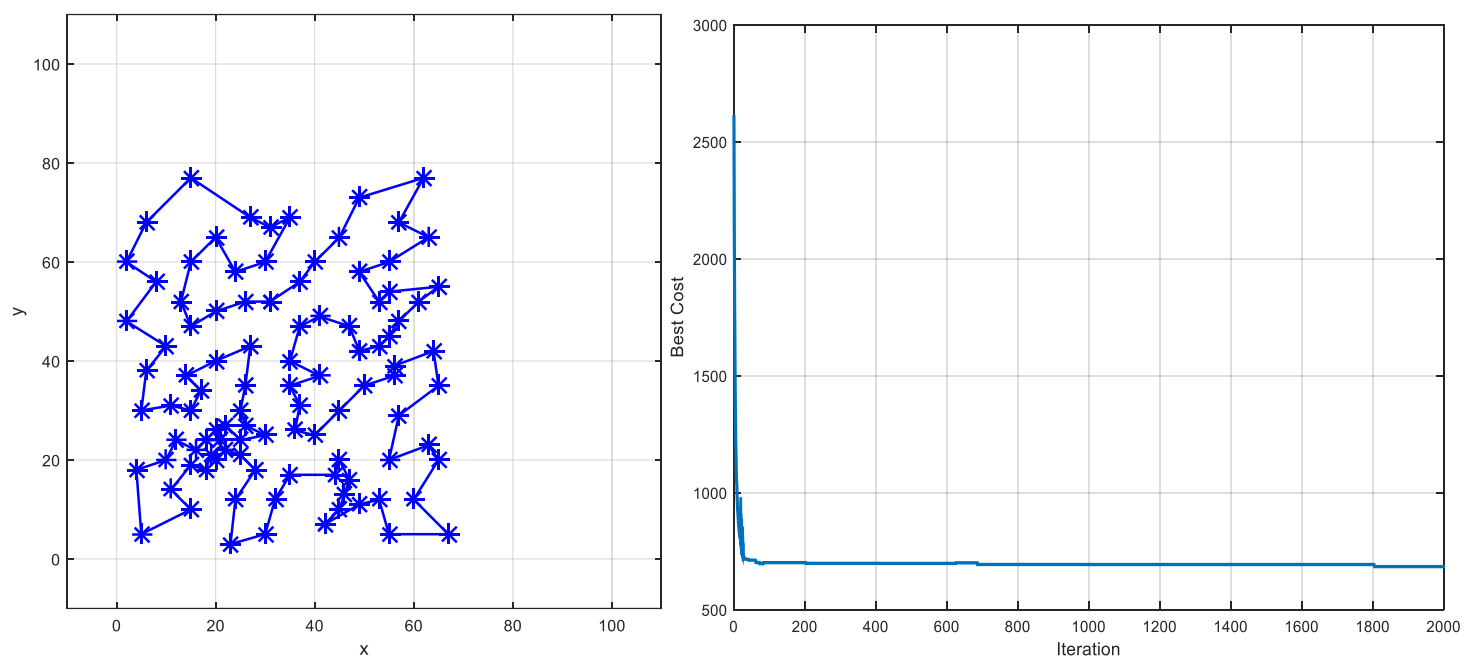

Fig.9. the result of 101-city (Eil101) TSPs using SOS-SA

\subsection{Descriptive Statistical analysis}

In this section, the Stata statistical package was used to further validate of the algorithm performance. The Shapiro-Will (W) test was used to formerly test whether the data were normally distributed. The Levene's test was used to test whether all the algorithms have the same variance, while both the oneway ANOVA test and Kruskal-Wallis test were used to test for difference in performance among all the algorithms. The oneway ANOVE is used whenever the parametric assumption were met, while the Kruskal-Wallis test is used whenever the parametric assumption were not met. In summary, descriptive statistics such as mean, standard deviation, minimum, maximum and range were used to describe the algorithms. Histograms and Shapiro-Wilk test were used to assess the normality of the algorithms, while the Levene's test was used to test the equality of Variance among the algorithms. Finally, to examine the significant difference in performance among the algorithms, the Friedman Test (with post hoc tests) was used. It is important to note that the difference in performance tests was done not based on the actual data but on transformed data. The main purpose of the difference in performance test was to verify that each of the selected algorithms that were compared with the SOS-SA is of high standard as claimed in the respective literatures. It is important to note that, in statistics, data transformation, which is the application of a deterministic mathematical function to each point in a data set, is utilized to help improve the normality of the experimental data sets and the interpretability or appearance of graphs (Zuur et al., 2010; Jason, 2002).

\subsubsection{Descriptive analysis of the SOS-SA and SOS algorithms}

Table 14 presents the descriptive statistics of the performance of SOS-SA and SOS with the theoretical value or the best known solution as the control algorithm. The SOS-SA is averagely smaller than the SOS in terms of mean, standard deviation, minimum, maximum and rang compared in terms of the best known solution. Therefore, this suggests that the SOS-SA is better than the SOS. The SOS' data has the widest range and spread 
of data around its mean value, while SOS-SA algorithm has the smallest range and dispersion of data around its mean value. Moreover, the standard deviations of the two algorithms are quiet high which suggests that there is great variation around the mean value for the two algorithms. The implication is that the data may not be normally distributed and parametric approaches cannot be used directly to test the significance of the difference in performance between SOS-SA and SOS.

Table 14: Descriptive statistics validation of SOS-SA and SOS algorithms compared to the best known solution (BKS)

\begin{tabular}{llllll}
\hline Algorithm & Mean & Std deviation & Min & Max & Range \\
\hline BKS & $29,546.60$ & $28,177.05$ & 426 & 96,772 & 96,346 \\
SOS & $30,545.45$ & $29,211.58$ & 429 & 98,018 & 97,589 \\
SOS_SA & $29,564.85$ & $28,223.54$ & 426 & 97,129 & 96,703 \\
\hline
\end{tabular}

To conveniently study whether the normality assumption is violated in the sample data under study, both informal and formal approaches were used. Based on the histograms (Fig. 10) of the algorithms SOS-SA and SOS, it can be readily seen that the algorithms' histograms are flatter and skewer than that of a normal distribution curve (Bell-shape curve) therefore providing more evidence of the non-normality of the three algorithms. However, the algorithms seem to follow the same form of skewness and flatness; meaning that, the spread of the data may not be significantly different within the two algorithms. In other words, the variance of the two algorithms may be equal.

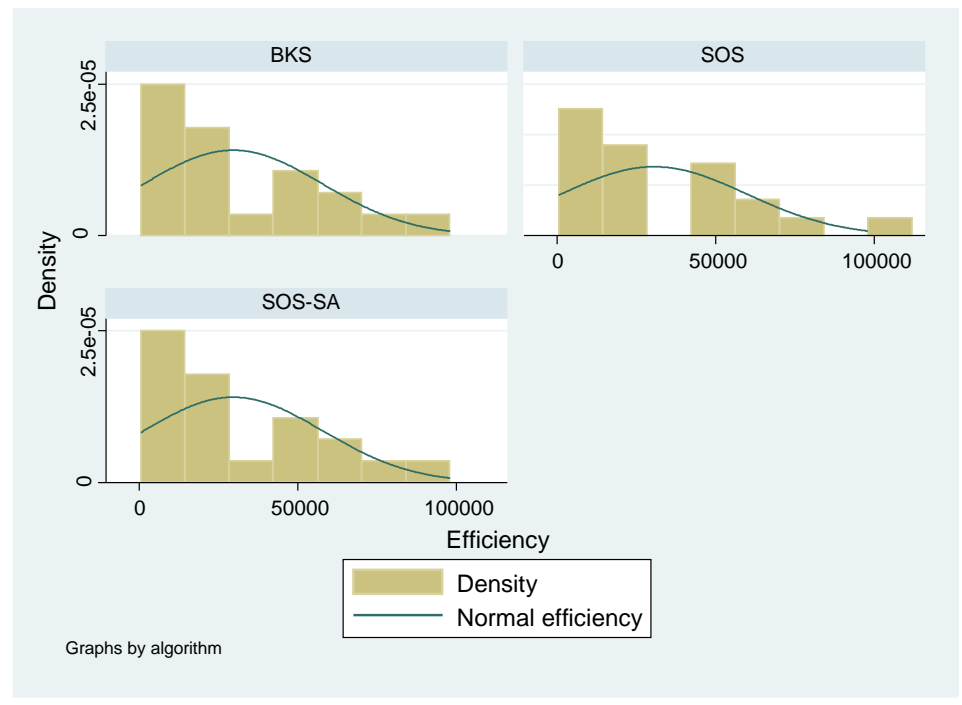

Figure 10: Histogram of SOS-SA and SOS-SA with the BKS as the control algorithm

Formally, the Shapiro-Wilk $(W)$ test statistic was considered in the analysis as being more robust than other tests statistics such as the skewness-Kurtosis, Kolmogorov-Smirnov and the Shapiro-Francia tests statistics. According to Table 15, after checking for the presence of outliers, the normality test was conducted based on the level data and four transformed data to avoid the problem of failing to adequately transform the data. The results show that the algorithms are indeed non-normally distributed at the level form, but were only found so based on the $\lambda$-parameter Box-Cox power transformation. On this basis, Box-Cox power transformed forms of the algorithms were further used for the test of equality of variance.

Table 15: Normality test of SOS-SA and SOS algorithms at different forms using the Shapiro-Wilk test statistic

\begin{tabular}{llll} 
Form & BKS & SOS & SOS-SA \\
\hline Level & $0.889^{* *}$ & $0.888^{* *}$ & $0.888^{* *}$ \\
Log & $0.813^{* * *}$ & $0.815^{* * *}$ & $0.812^{* * *}$ \\
Square root & $0.813^{* * *}$ & $0.815^{* * *}$ & $0.812^{* * *}$ \\
K-parameter log & $0.895^{* * *}$ & $0.893^{* * *}$ & $0.895^{* * *}$ \\
Lambda-parameter Box-Cox power & 0.907 & 0.905 & 0.906 \\
\hline
\end{tabular}

Note: $* * *<0.01$ and $* *<0.05$ 
The analysis of equal variance across the three algorithms was based on the Levene's test because it is robust even with departure from normality of the data. The result in Table 16 indicates that based on mean, median and $10^{\text {th }}$ percentile, the test was found to be statistically insignificant; implying that, the null hypothesis of equal variance cannot be rejected. In other words, compared with the best known solution (BKS), SOS-SA and SOS have equal variance.

Table 16: Equal variance test of SOS-SA and SOS algorithms against the best known solution using the Levene's test statistic

\begin{tabular}{lll} 
Statistic & Levene's & P-value \\
\hline W0 & 0.001 & 0.998 \\
W50 & 0.001 & 0.998 \\
W10 & 0.002 & 0.997 \\
\hline
\end{tabular}

$W 0=$ mean, $W 50=$ Median, $W 10=10^{\text {th }}$ percentile.

Based on the findings obtained so far, one way Analysis of Variance (ANOVA) was carried out to assess the difference between the BKS, SOS-SA and SOS. The result of the test presented in Table 17 indicates that the majority of variation in the algorithms' observations is explained in the variation within and not the variation between. In other words, the contribution of the model to explain the difference between the three methods is minimal in relation to that of the residual. Consequently, the ANOVA test was found to be statistically insignificant on the basis of the low F-statistic and high p-value. In other words, there is no statistically significant difference between the best known solution and the proposed SOS-SA algorithm.

Table 17: Oneway ANOVA test of the difference between SOS-SA and SOS compared to the best known solution

\begin{tabular}{llllll}
\hline Source of variation & SS & df & MS & F & P-value \\
\hline Between group & 250.82 & 2 & 125.41 & $2.30 \mathrm{E}-03$ & 0.997 \\
Within group & $3,106,466.42$ & 57 & $54,499.41$ & & \\
Total & $3,106,717.25$ & 59 & 52656.22 & & \\
\hline
\end{tabular}

\subsubsection{Descriptive analysis of the GA-PSO-ACO, MSA-IBS, LBSA, and SOS-SA algorithms}

Table 18 presents the descriptive statistics of the performance of four algorithms namely, GA-PSO-ACO, MSAIBS, LBSA, and SA- SOS with the best known solution as the control algorithm. The SOS-SA algorithm is averagely the smallest algorithm followed by LBSA, suggesting that SOS-SA is the best algorithm followed by LBSA. The standard deviations of all the algorithms are quite high suggesting that there is great variation around the mean value for all the algorithms. In other words, the data may not be normally distributed and parametric approaches cannot be used directly to test the significance of the difference among the algorithms.

\begin{tabular}{lllllll} 
Table 18: Descriptive statistics of GA-PSO-ACO, LBSA, MSA-IBS, and SOS-SA to the best kno \\
\cline { 2 - 6 } & Algorithm & Mean & Std dev. & Min & Max & Range \\
\hline & GA-PSO-ACO & $2,063,992$ & $11,900,000$ & 426 & $70,300,000$ & $70,299,574$ \\
LBSA & $1,997,597$ & $11,500,000$ & 426 & $68,000,000$ & $67,999,574$ \\
MSA-IBS & $1,993,722$ & $11,500,000$ & 426 & $67,900,000$ & $67,899,574$ \\
SOS-SA & $1,990,455$ & $11,400,000$ & 426 & $67,800,000$ & $67,799,574$ \\
BKS & $1,941,301$ & $11,200,000$ & 426 & $66,000,000$ & $65,999,574$ \\
\hline
\end{tabular}

Based on the histograms (Fig. 11) of the algorithms GA-PSO-ACO, LBSA, MSA-IBS, and SOS-SA, it can be readily be seen that the algorithms do not follow a normal distribution therefore providing more evidence of the non-normality of the data. 


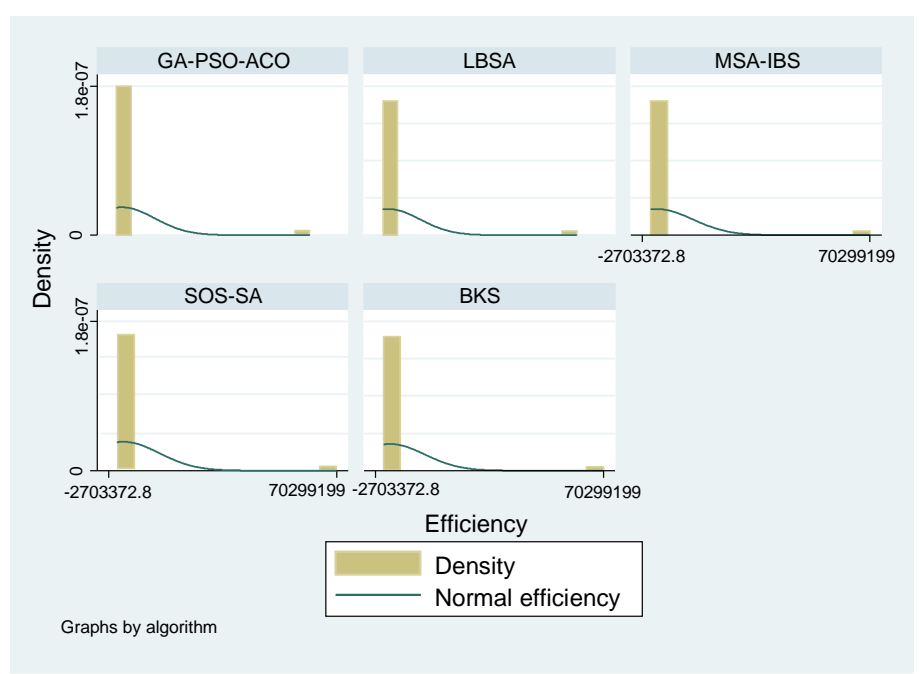

Fig. 11: Histogram of GA-PSO-ACO, LBSA, MSA-IBS with BKS as the control algorithm

According to Table 19, after checking for the presence of outliers, the normality test was conducted based on the level data and four transformed data to avoid the problem of failing to adequately transform the data. The results show that the algorithms are indeed non-normally distributed even after various transformations. The test of the difference among the four (4) algorithms based on their transformed data was carried out using the Kruskal-Wallis test.

Table 19: Normality test for GA-PSO-ACO, LBSA, MSA-IBS, and SOS-SA algorithms at different forms using the Shapiro-Wilk test

\begin{tabular}{llllll}
\hline Form & GA-PSO-ACO & LBSA & MSA-IBS & SOS-SA & BKS \\
\hline Level & $0.16557^{* * *}$ & $0.16564^{* * *}$ & $0.16564^{* * *}$ & $0.16563^{* * *}$ & $0.1657^{* * *}$ \\
Log & $0.90744^{* * *}$ & $0.90755^{* * *}$ & $0.90754^{* * *}$ & $0.90756^{* * *}$ & $0.90775^{* * *}$ \\
Square root & $0.90744^{* * *}$ & $0.90755^{* * *}$ & $0.90754^{* * *}$ & $0.90756^{* * *}$ & $0.90775^{* * *}$ \\
K-parameter log & $0.9189^{* *}$ & $0.91886^{* *}$ & $0.91886^{* *}$ & $\mathrm{Na}$ & $\mathrm{Na}$ \\
Lambda-parameter Box-Cox power & $\mathrm{Na}$ & $\mathrm{Na}$ & $\mathrm{Na}$ & $\mathrm{Na}$ & $\mathrm{Na}$ \\
\hline
\end{tabular}

Note: $* * *<0.01$

The Kruskal-Wallis test is a nonparametric test that does not depend on the normality and homogeneity of the data. The analysis is based not on the original data but on the rank of the data after sorting them in ascending order. In Table 20, the result of the test both with and without ties indicates that based on the transformed data, there is no statistically significant difference among SOS-SA, LBSA, and MSA-IBS and that the difference observe could be due to sampling peculiarity. This is also to verify that SOS-SA is being compared with some of the best state-of-the-art heuristic algorithms, having the most similar techniques of implementation with the SOS-SA.

Table 20: Difference analysis of GA-PSO-ACO, LBSA, MSA-IBS, SOS-SA and BKS using Kruskal-Wallis test

\begin{tabular}{llll} 
& & \multicolumn{2}{c}{ Chi-squared } \\
\cline { 3 - 4 } Algorithm & Sum Rank & Without ties & With ties \\
\hline GA-PSO-ACO & 3,162 & 0.107 & 0.107 \\
LBSA & 3,069 & & \\
MSA-IBS & $3,079.5$ & & \\
SOS-SA & 3,046 & & \\
BKS & $3,033.5$ & & \\
\hline
\end{tabular}

\subsubsection{Descriptive analysis of SOS-SA and IBA algorithms}

Table 21 presents the descriptive statistics of the performance of SOS-SA and IBA compared with the best known solution. The SOS-SA algorithm is averagely the smallest, therefore, this suggest that SOS-SA performs 
better than the IBA. The IBA's data has the widest range and spread of data around its mean value while SOSSA has the smallest range and dispersion of data around its mean value compared to the best known solution. Moreover, the standard deviations of the two algorithms are quiet high which suggests that there is great variation around the mean value for all the algorithms. The implication is that the data may not be normally distributed and parametric approaches cannot be used directly to test the significance of the difference in performance among SOS-SA and IBA.

\begin{tabular}{lccccc}
\multicolumn{6}{c}{ Table 21: Descriptive statistics of IBA, BKS and SOS_SA } \\
\hline Algorithm & Mean & Std deviation & Min & Max & Range \\
\hline IBA & $31,277.69$ & $29,494.45$ & 426 & 97,547 & 97,121 \\
BKS & $31,175.13$ & $29,330.33$ & 426 & 96,772 & 96,346 \\
SOS-SA & $31,199.25$ & $29,384.09$ & 426 & 97,129 & 96,703 \\
\hline
\end{tabular}

Based on the histograms (Fig. 12) of the two algorithms, it can be readily seen that the algorithms' histograms are flatter and skewer than that of a normal distribution curve (Bell-shape curve) therefore providing more evidence of the non-normality of the two algorithms. However, both algorithms with the BKS seem to follow the same form of skewness and flatness; meaning that, the spread of the data may not be significantly different within the three algorithms. In other words, the variance of the three algorithms may be equal.

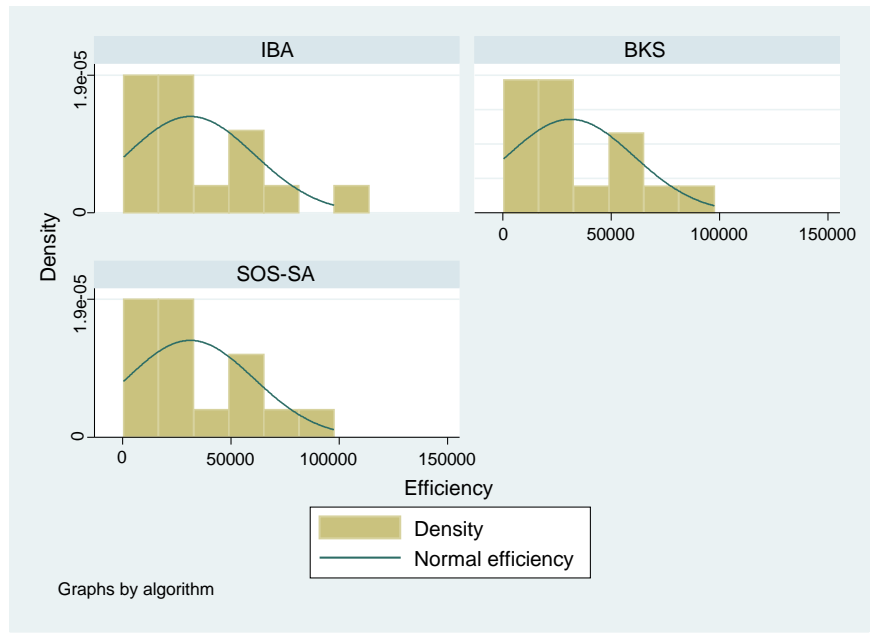

Fig. 12: Histogram of SOS-SA and IBA with BKS as the control algorithm

According to Table 22, the results show that the algorithms are indeed non-normally distributed at the level form but were only found so based on the $\lambda$-parameter Box-Cox power transformation. On this basis, Box-Cox power transformed forms of the algorithms were further used for the test of equality of variance.

\begin{tabular}{lllc} 
Table 22: Normality test of BKS, SOS_SA, and IBA algorithms at different forms using the Shapiro-Wilk test statistic \\
\hline Form & BKS & IBA & SOS_SA \\
\hline Level & $0.8901^{*}$ & $0.8894^{*}$ & $0.8901^{*}$ \\
Log & $0.8058^{* * *}$ & $0.8065^{* * *}$ & $0.806^{* * *}$ \\
Square root & $0.8058^{* * *}$ & $0.8065^{* * *}$ & $0.806^{* * *}$ \\
K-parameter log & $0.9123^{* *}$ & $0.9123^{* *}$ & $0.9124^{* *}$ \\
Lambda-parameter Box-Cox power & 0.9218 & 0.9219 & 0.9219 \\
\hline
\end{tabular}

Note: $* * * 0.01$ and $* *<0.05$

The analysis of equal variance across the two algorithms was based on the Levene's test because it is robust even with departure from normality of the data. The result in Table 23 indicates that based on mean, median and $10^{\text {th }}$ percentile, the test was found to be statistically insignificant; implying that, the null hypothesis of equal variance cannot be rejected. In other words, SOS-SA and IBA, with the best known solution have equal variance. 
Table 23: Equal variance test of SOS-SA and IBA algorithms to the best known solution using the Levene's test statistic

\begin{tabular}{lll}
\hline Statistic & Levene's & P-value \\
\hline W0 & 0.0053 & 0.9946 \\
W50 & 0.0048 & 0.9951 \\
W10 & 0.0051 & 0.9949 \\
\hline
\end{tabular}

W0=mean, $W 50=$ Median, $W 10=10$ th percentile.

Based on the findings obtained so far, one-way Analysis of Variance (ANOVA) was carried out to assess the difference between SOS-SA and IBA algorithms with the best known solution as the control algorithm. The result of the test presented in Table 24 indicates that the majority of variation in the algorithms' observations is explained the variation within and not the variation between. In other words, the contribution of the model to explain the difference between the three algorithms is minimal in relation to that of the residual. Consequently the ANOVA test was found to be statistically insignificant on the basis of the low F-statistic and high p-value. In other words, there is no statistically significant difference between the two algorithms.

\begin{tabular}{llllll}
\multicolumn{6}{l}{ Table 24: Oneway ANOVA test of the difference between SOS-SA and IBA } \\
\hline Source of variation & SS & df & MS & F & P-value \\
\hline Between group & 643.66 & 2 & 321.83 & 0.01 & 0.9943 \\
Within group & $2,519,515.64$ & 45 & $55,989.24$ & & \\
Total & $2,520,159.30$ & 47 & 53620.41 & & \\
\hline
\end{tabular}

Note: $\mathrm{SS}=$ Sum of Squares, $\mathrm{MS}=$ Mean Sum of Square, $\mathrm{df}=$ Degree of freedom

\subsubsection{Friedman Test (with post hoc tests) analysis of algorithm performance}

In this section, the Friedman's non-parametric test was further used to check for existence of any significant difference in performance among the algorithms whilst running, $\chi^{2}(1)=19, p$-value $=0.000$ for comparison between SOS-SA and SOS, $\chi^{2}(1)=8, p$-value $=0.005$ for comparison between SOS-SA and IBA, and $\chi^{2}(3)=$ $73.189, p$-value $=0.000$ for comparison among SOS-SA with GA-PSO-ACO, MSA-IBS, and LBSA. The median (IQR) perceived effort levels for the SOS and SOS-SA running trial were 21681 (848 to 51013) and 21681 (812 to 48956), while the median (IQR) perceived effort levels for the IBA and SOS-SA running trial were 21681 (2392 to 56341) and 21681 (2391 to 56205), respectively. It can be concluded that there are statistically significant differences among the algorithms based on the mean ranking returned by the Friedman's test presented in table 25. However, since the Friedman's test can only show the existence of significant difference between two algorithms, the test does not pinpoint which groups in particular differ from each other in the case of multiple algorithms comparison (for example, GA-PSO-ACO, MSA-IBS, LBSA, and SOS-SA). Therefore, to adequately evaluate the statistical performance of the SOS-SA, the Friedman test with post hoc tests was further conducted and the result obtained is as reported next.

\begin{tabular}{llllll}
\multicolumn{2}{c}{ Table 25: Mean ranking returned by Friedman's non parametric test } \\
\hline \multicolumn{2}{c}{ Test 1 } & \multicolumn{2}{c}{ Test 2 } & \multicolumn{2}{c}{ Test 2 } \\
Algorithms & Ranking & Algorithms & Ranking & Algorithms & Ranking \\
\hline GA-PSO-ACO & 3.91 & SOS & 2.00 & IBA & 1.75 \\
MSA-IBS & 2.24 & SOS-SA & 1.00 & SOS-SA & 1.25 \\
LBSA & 1.96 & & & & \\
SOS-SA & 1.89 & & & & \\
\hline
\end{tabular}

The Friedman test with post hoc tests indicate that there was a statistically significant difference in the performance of SOS-SA with GA-PSO-ACO, MSA-IBS, and LBSA, this is observed whilst running, $\chi^{2}(3)=$ $73.189, p$-value $=0.000$. Post hoc analysis with Wilcoxon signed-rank tests was conducted with a Bonferroni correction applied, resulting in a significance level set at computed $p$-value $<0.0125$ (i.e. $0.05 / 4$, since we are comparing GA-PSO-ACO, MSA-IBS, LBSA, and SOS-SA). The median (IQR) perceived effort levels for each of the GA-PSO-ACO, MSA-IBS, LBSA, and SOS-SA running trial were 33524 (6912 to 59051), 33522 (6819 to 59030), 33522 (6829 to 59030), and 33523 (6773 to 58985) respectively. There were statistically significant differences between the performance of SOS-SA and GA-PSO-ACO running trials $(\mathrm{Z}=-5.012, p$-value $=$ 0.000). However, there were no significant differences between the SOS-SA and MSA-IBS running trials $(\mathrm{Z}=$ $1.625, p$-value $=0.104)$, or between SOS-SA and LBSA running trials $(Z=-0.355, p$-value $=0.722)$.

In summary, the statistical analysis has revealed some interesting results with respect to all the algorithms that were compared with the proposed optimization method. First, the analysis result showed that the SOS-SA 
performed favourable well compared to other state-of-the-art algorithms. This is verified based on the result of the descriptive statistics test using Mean, Standard deviation, Min, Max, and Range described in Tables 14, 18 and 21 respectively. This also corresponds to the analysis of the results presented in Tables 11,12 , and 13, were SOS-SA (with 32\%) outperformed the other three algorithms namely, GA-PSO-ACO (with 19\%), MSA-IBS (with 20\%), and LBSA (with 28\%) in terms of convergence. Similar evaluation with SOS and IBA showed the convergence performance of SOS-SA to be $95 \%$ (19 out 20 instances) and 50\% (8 out of 16 instances) for both SOS and IBA. Second, based on the performance difference among all the algorithms compared with SOS-SA using transformed data and with BKS as control algorithm, the analysis revealed that MSA-IBS, LBSA, and IBA are equally good algorithms as claimed by the respective authors. Third, SOS-SA appears to be next to the control algorithm (or BKS) in most of the performance analysis result presented, for instance in Table 20, the Sum Rank (i.e., SOS-SA $=3,046$ and BKS = 3,033.5). Finally, considering the Friedman Test (with post hoc tests) analysis for the individual algorithm performances, since the $p$-values of GA-PSO-ACO, SOS, and IBA are less than 0.05 , we can say that SOS-SA is statistically significantly better. In the case of MSA-IBS and LBSA, since their p-values are greater than the computed p-value of 0.0125 , therefore, we can say that there are no significant differences in performance between these algorithms and the SOS-SA. Therefore, this consequently verifies the initial claim that the SOS-SA algorithm can compete favorable with even the best known solution, as it tends to perform better in some cases than the BKS, which then verifies the results presented in Table 2. The unique advantage of the SOS-SA over other algorithms can be attributed to its capability to deeply explore and exploit problem search space, during search process. This is made possible by the benefit factor mechanism in the mutualism phase and the artificial vector mechanisms in the parasitism phase of the SOS. Finally, we conclude this analysis section by saying that the proposed SOS-SA optimization method has promising and immense potential for solving the TSP as well as other complex discrete problems.

\subsection{Remarks}

In the course of the empirical evaluation of the proposed algorithms, some potential Challenges were observed, which are highlighted as follows:

- Computation Time. Though the SOS-SA algorithm performed favourably against the best known available solution from TSPLIB and other state-of-the-art algorithms, there is still room for improvement in terms of computational time, and more specifically, the iterative computation of tour cost function. The SOS-SA algorithm spends time recalculating the cost function with every change in iteration and most importantly, it was also observed that the computation cost increases proportionately with increase in the dimension of the TSP problem instance. A more simplified and adaptive method of calculating the cost function is therefore required to speed-up the computation time.

- Acceptance probability function. Similarly, the computation of the acceptance probability function consumes a lot of system resources, more specifically, CPU time. This is as a result of the exponential computation required to determine the probability of acceptance or rejection of a new solution. Therefore, approximating the calculation of this function without compromising the decision rule can significantly improve the performance of the framework in terms of cost of execution.

- SA parameter selection. SA parameter required some level of experience in selecting a good set of performance parameters, as they would partly affect the performance of the SOS-SA algorithm, in escaping global minimum as quickly as possible. Selecting a good set of SA performance parameters was the main bottleneck experienced during the simulation experiment, as parameter fine-tuning were made more frequently. This challenge also affected the length of time the optimal solutions were attained. A typical example is the selection of an appropriate cooling schedule for the different simulations. Finding an appropriate cooling schedule was a major challenge for the SOS-SA implementation from one problem instance to another. One possible solution is to implement an adaptive method of setting the cooling schedule for different problem instances.

- Scalability. In the course of the simulation process specifically for large length TSP problems, the system ran out of memory severally as the dimension of the TSPLIB benchmark increased, as it was observed for Pla33810 and Pla85900. These two instances, during execution, required additional memory. Therefore, considering also the first two aforementioned issues, one possible option would be to identify possible parallelism for the SOS-SA algorithm, which can improve both framework computational time and memory utilization concurrently.

\section{Conclusion and Future Direction}

In this paper, a novel and hybrid simulated annealing based symbiotic organisms search algorithm is proposed as a new approach for solving symmetric TSP. The SOS algorithm which is inspired by the symbiotic relationships among organisms in the ecosystem was initially proposed to handle engineering optimization 
problems. The design of a hybrid SOS-SA framework, which incorporates the SA local search capability into the problem search space of SOS algorithm, and the application of the simulation results of the SOS-SA to the TSP were discussed. The simulation results supports the fact that the new SOS-SA framework can realise TSP optimal solutions and compete favourably with other state-of-the-art optimization algorithms being applied to the TSP related problems and complex discrete problems. As future work the authors intend to further improve the algorithm by testing its scalability in a parallel and distributed environments for various Big Data graphs from SNAP (https://snap.stanford.edu/data/) with different properties (e.g., sparse, dense, power law). Scalability will be tested when problem size is fixed and number of cores/machines increases, and when the number of machines is fixed and the problem increases.

\section{Funding}

This work was supported by the research grant from University of KwaZulu-Natal, College of Agriculture, Engineering and Science. Durban, South Africa.

\section{Author Contributions}

AEE and AOA conceived and designed the experiments, AEE and AOA performed the experiments, AEE and MEF analysed the data, AEE, AOA and MEF wrote the paper.

\section{Conflict of Interest}

The authors declare that there is no conflict of interests regarding the publication of this paper.

\section{Reference}

Applegate, D. L., Bixby, R. E., Chvatal, V., \& Cook, W. J. (2011). The traveling salesman problem: a computational study. Princeton university press.

Aulady, M. (2013). A hybrid symbiotic organisms search-quantum neural network for predicting high performance concrete compressive strength.

Barbato, M., Grappe, R., Lacroix, M., \& Calvo, R. W. (2016). Polyhedral results and a branch-and-cut algorithm for the double traveling Salesman problem with multiple stacks. Discrete Optimization, 21, 25-41.

Bellman, R. (1962). Dynamic programming treatment of the travelling salesman problem. Journal of the ACM (JACM), 9(1), pp.61-63.

Bender, M. A., \& Chekuri, C. (2000). Performance guarantees for the TSP with a parameterized triangle inequality. Information Processing Letters, 73(1), 17-21.

Chen, S. M., \& Chien, C. Y. (2011). Solving the traveling salesman problem based on the genetic simulated annealing ant colony system with particle swarm optimization techniques. Expert Systems With Applications, 38(12), 14439-14450.

Cheng, M. Y, Prayogo, D. (2014). Symbiotic organism search: A new metaheuristic optimization. Computers and Structures, 139:98-112.

Cheng, M.Y., Prayogo, D. and Tran, D. H. (2015). Optimizing multiple-resources levelling in multiple projects using discrete symbiotic organisms search. Journal of Computing in Civil Engineering, 30(3), p.04015036.

Cornu, M., Cazenave, T., \& Vanderpooten, D. (2016). Perturbed Decomposition Algorithm applied to the multiobjective Traveling Salesman Problem. Computers \& Operations Research.

Çunkaş, M. and Özsağlam, M. Y. (2009). A comparative study on particle swarm optimization and genetic algorithms for traveling salesman problems. Cybernetics and Systems: An International Journal, 40(6), pp.490-507.

Delgadillo, F. J. D., Montiel, O., \& Sepúlveda, R. (2016). Reducing the size of traveling salesman problems using vaccination by fuzzy selector. Expert Systems with Applications, 49(1), 20-30.

Deng, W., Chen, R., He, B., Liu, Y., Yin, L. and Guo, J. (2012). A novel two-stage hybrid swarm intelligence optimization algorithm and application. Soft Computing, 16(10), pp.1707-1722.

Dorigo, M. and Gambardella, L. M. (1997). Ant colonies for the travelling salesman problem. BioSystems, 43(2), pp.73-81.

Durbin, R. (1987). An analogue approach to the travelling salesman. Nature, 326, p.16.

Durbin, R., Szeliski, R. and Yuille, A. (1989). An analysis of the elastic net approach to the traveling salesman problem. Neural Computation, 1(3), pp.348-358.

Fang, L., Chen, P., \& Liu, S. (2007). Particle swarm optimization with simulated annealing for TSP. In Proceedings of the 6th WSEAS International Conference on Artificial Intelligence, Knowledge Engineering and Data Bases (AIKED’07) (pp. 206-210). 
Farmer, J. D., Packard, N. H. and Perelson, A. S. (1986). The immune system, adaptation, and machine learning. Physica D: Nonlinear Phenomena, 22(1), pp.187-204.

Feng, X., Lau, F.C. and Gao, D. (2009). A New Bio-inspired Approach to the Traveling Salesman Problem. Complex Sciences, pp.1310-1321.

Garey, M. R., \& Johnson, D. S. (1979). Com-puters and Intractablility: A Guide to the Theory of NPCompleteness.

Geng, X., Chen, Z., Yang, W., Shi, D. and Zhao, K. (2011). Solving the traveling salesman problem based on an adaptive simulated annealing algorithm with greedy search. Applied Soft Computing, 11(4), pp.36803689.

Jason, O. (2002). Notes on the use of data transformations. Practical Assessment, Research \& Evaluation, 8(6).

Johnson, D. S. (1990). Local optimization and the traveling salesman problem. In International Colloquium on Automata, Languages, and Programming (pp. 446-461). Springer Berlin Heidelberg.

Johnson, D. S. and McGeoch, L. A. (1997). The traveling salesman problem: A case study in local optimization. Local search in combinatorial optimization, 1, pp.215-310.

Jolai, F., and Ghanbari, A. (2010). Integrating data transformation techniques with Hopfield neural networks for solving travelling salesman problem. Expert Systems with Applications, 37(7), 5331-5335.

Jünger, M., Reinelt, G., \& Rinaldi, G. (1995). The traveling salesman problem. Handbooks in operations research and management science, 7, 225-330.

Kanda, J., de Carvalho, A., Hruschka, E., Soares, C., \& Brazdil, P. (2016). Meta-learning to select the best metaheuristic for the Traveling Salesman Problem: A comparison of meta-features. Neurocomputing.

Katayama, K., Sakamoto, H., \& Narihisa, H. (2000). The efficiency of hybrid mutation genetic algorithm for the travelling salesman problem. Mathematical and Computer Modelling, 31(10), 197-203.

Kirkpatrick, S., Gelatt, C. D. and Vecchi, M. P. (1983). Optimization by simulated annealing. science, 220(4598), pp.671-680.

Knox, J. (1994). Tabu search performance on the symmetric traveling salesman problem. Computers \& Operations Research, 21(8), pp.867-876.

Lawler, E.L. and Wood, D.E. (1966). Branch-and-bound methods: A survey. Operations research, 14(4), pp.699-719.

Lin, Y., Bian, Z., \& Liu, X. (2016). Developing a dynamic neighborhood structure for an adaptive hybrid simulated annealing-tabu search algorithm to solve the symmetrical traveling salesman problem. Applied Soft Computing, 49, 937-952.

Lourenço, H. R., Martin, O. C. and Stützle, T. (2003). Iterated local search. In Handbook of metaheuristics (pp. 320-353). Springer US.

Malek, M., Guruswamy, M., Pandya, M., \& Owens, H. (1989). Serial and parallel simulated annealing and tabu search algorithms for the traveling salesman problem. Annals of Operations Research, 21(1), 59-84.

Matai, R., Singh, S. P. and Mittal, M. L. (2010). Traveling salesman problem: An overview of applications, formulations, and solution approaches. Traveling Salesman Problem, Theory and Applications, pp.1-24.

Mohan, U., Ramani, S., \& Mishra, S. (2016). Constant factor approximation algorithm for TSP satisfying a biased triangle inequality. Theoretical Computer Science, 657, 111-126.

Osaba, E., Yang, X. S., Diaz, F., Lopez-Garcia, P., \& Carballedo, R. (2016). An improved discrete bat algorithm for symmetric and asymmetric Traveling Salesman Problems. Engineering Applications of Artificial Intelligence, 48, 59-71.

Ouaarab, A., Ahiod, B., \& Yang, X. S. (2014). Improved and discrete cuckoo search for solving the travelling salesman problem. In Cuckoo Search and Firefly Algorithm (pp. 63-84). Springer International Publishing.

Ozcan, E. and Erenturk, M. (2004). A brief review of memetic algorithms for solving Euclidean 2D traveling salesrep problem. In Proc. of the 13th Turkish Symposium on Artificial Intelligence and Neural Networks (pp. 99-108).

Reinelt, G. (1994). The traveling salesman: computational solutions for TSP applications. Springer-Verlag.

Shi, X. H., Liang, Y. C., Lee, H. P., Lu, C. and Wang, Q. X. (2007). Particle swarm optimization-based algorithms for TSP and generalized TSP. Information Processing Letters, 103(5), pp.169-176.

Sundar, K., \& Rathinam, S. (2016). Generalized multiple depot traveling salesmen problem-Polyhedral study and exact algorithm. Computers \& Operations Research, 70, 39-55.

Talbi, E. G. (2002). A taxonomy of hybrid metaheuristics. Journal of heuristics, 8(5), 541-564.

Tran, D. H., Cheng, M. Y. and Prayogo, D. (2016). A novel Multiple Objective Symbiotic Organisms Search (MOSOS) for time-cost-labor utilization tradeoff problem. Knowledge-Based Systems, 94, pp.132-145.

Tsai, C. F., Tsai, C. W., \& Tseng, C. C. (2004). A new hybrid heuristic approach for solving large traveling salesman problem. Information Sciences, 166(1), 67-81.

Verma, S., Saha, S., and Mukherjee, V. (2015). A novel symbiotic organisms search algorithm for congestion management in deregulated environment. Journal of Experimental \& Theoretical Artificial Intelligence, $1-21$. 
Vincent, F. Y., Redi, A. P., Yang, C. L., Ruskartina, E., \& Santosa, B. (2016). Symbiotic organisms search and two solution representations for solving the capacitated vehicle routing problem. Applied Soft Computing.

Volgenant, T. and Jonker, R. (1982). A branch and bound algorithm for the symmetric traveling salesman problem based on the 1-tree relaxation. European Journal of Operational Research, 9(1), pp.83-89.

Wang, C., Lin, M., Zhong, Y. and Zhang, H. (2015). Solving travelling salesman problem using multiagent simulated annealing algorithm with instance-based sampling. International Journal of Computing Science and Mathematics, 6(4), pp.336-353.

Wang, J., Ersoy, O. K., He, M., \& Wang, F. (2016). Multi-offspring genetic algorithm and its application to the traveling salesman problem. Applied Soft Computing, 43, 415-423.

Zhan, S. H., Lin, J., Zhang, Z. J. and Zhong, Y. W. (2016). List-Based Simulated Annealing Algorithm for Traveling Salesman Problem. Computational intelligence and neuroscience, 2016, pp. 1-12.

Zhang, H., \& Zhou, J. (2016). Dynamic multiscale region search algorithm using vitality selection for traveling salesman problem. Expert Systems with Applications, 60, 81-95.

Zhang, H., Tong, W., Xu, Y., \& Lin, G. (2016). The Steiner traveling salesman problem with online advanced edge blockages. Computers \& Operations Research, 70, 26-38.

Zhou, Y., Luo, Q., Chen, H., He, A. and Wu, J., 2015. A discrete invasive weed optimization algorithm for solving traveling salesman problem. Neurocomputing, 151, pp.1227-1236.

Zuur, A. F., Ieno, E. N., \& Elphick, C. S. (2010). A protocol for data exploration to avoid common statistical problems. Methods in Ecology and Evolution, 1(1), 3-14.

Appendix 1. Simulation results demonstrating the convergence curves for Pr107, Pr124, U159, Rat195, Gi1262, Pr299, Pcb442, and Rat575 TSPLIB instance.

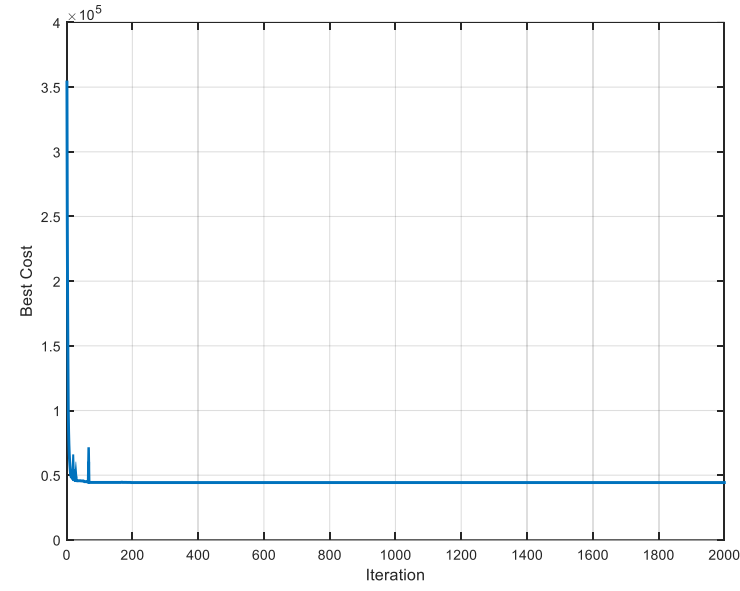

Convergence curve for $\operatorname{Pr} 107$

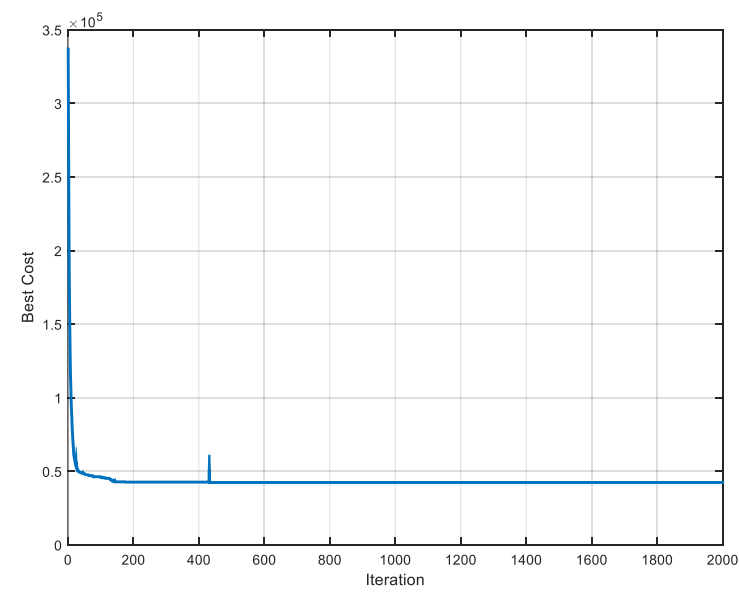

Fig.3A. Convergence curve for U159

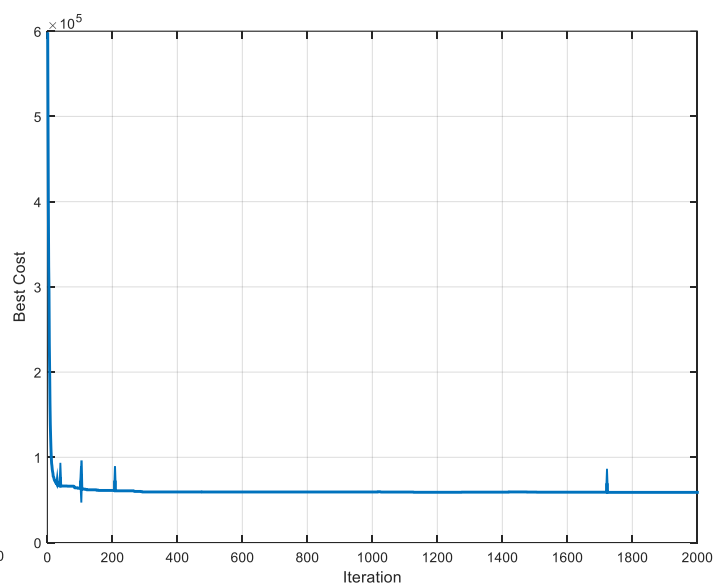

Fig.A2. Convergence curve for Pr124

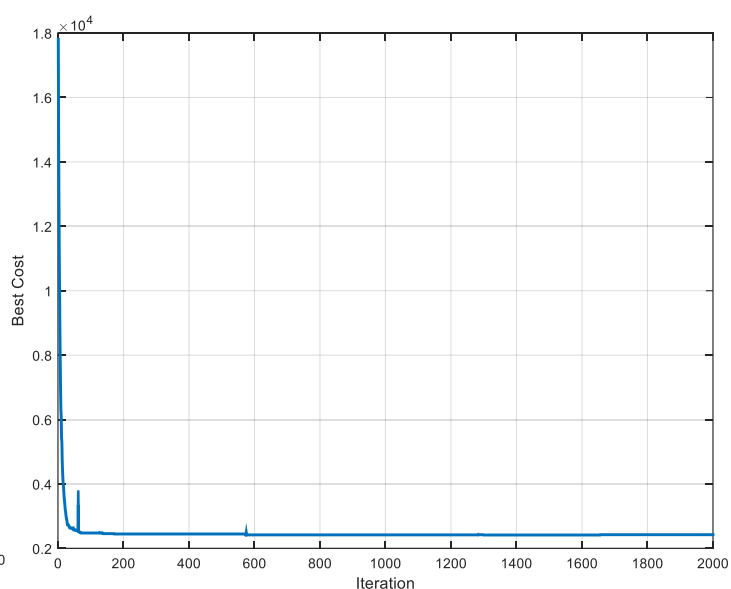

Fig.4A. Convergence curve for Rat195 


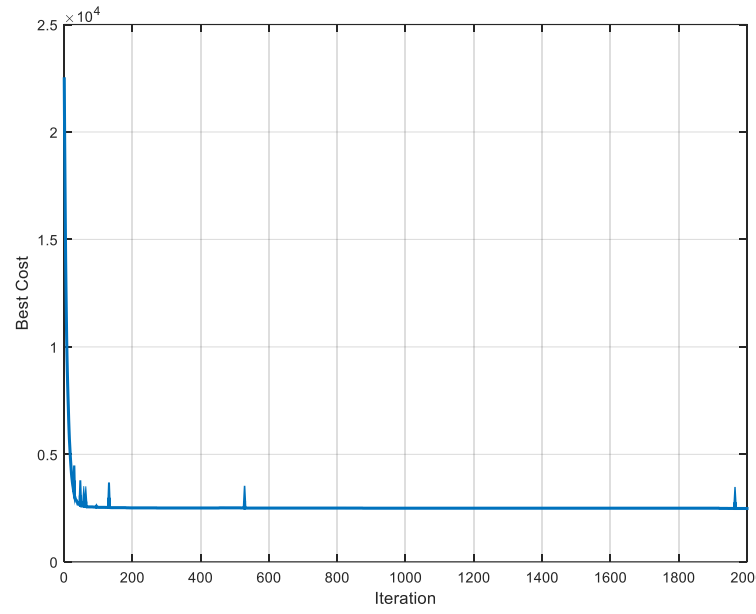

Fig.5A. Convergence curve for Gil262

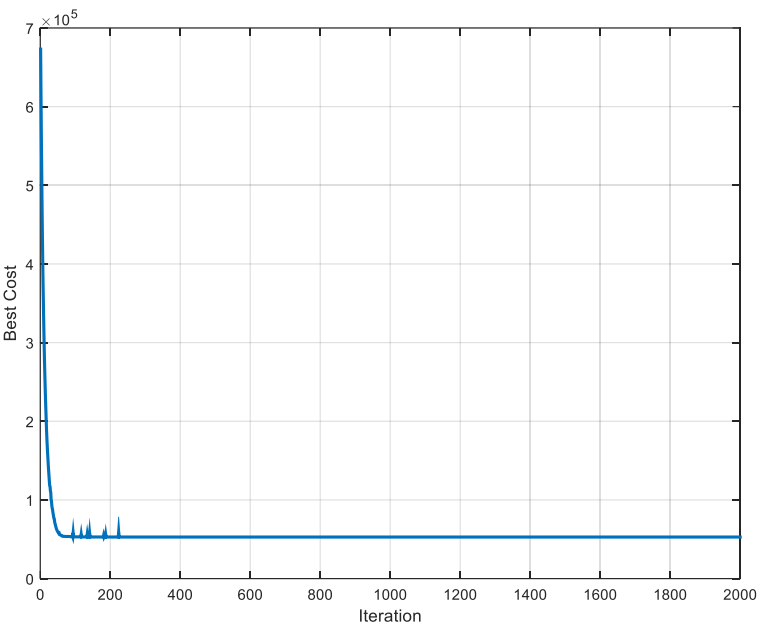

Fig.7A. Convergence curve for Pcb442

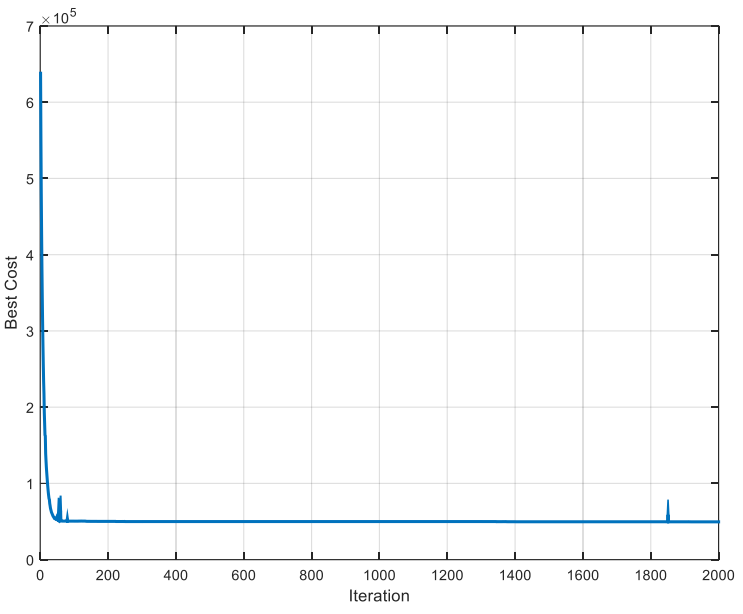

Fig.6A. Convergence curve for Pr299

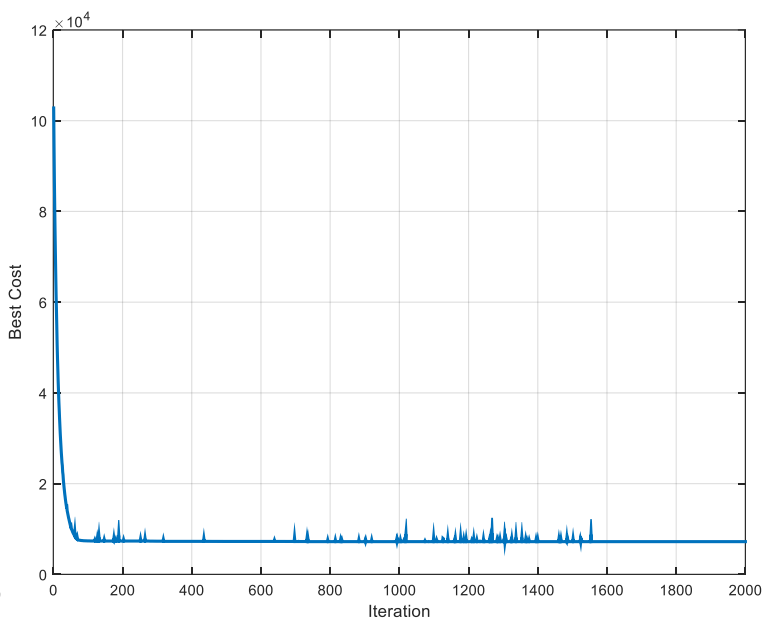

Fig.8A. Convergence curve for Rat575 